\title{
CONNECTIONS ON PARAHORIC TORSORS OVER CURVES
}

\author{
VIKRAMAN BALAJI, INDRANIL BISWAS, AND YASHONIDHI PANDEY
}

\begin{abstract}
Aвstract. We define parahoric $\mathscr{G}$-torsors for certain Bruhat-Tits group scheme $\mathscr{G}$ on a smooth complex projective curve $X$ when the weights are real, and also define connections on them. We prove that a $\mathscr{G}$-torsor is given by a homomorphism from $\pi_{1}(X \backslash D)$ to a maximal compact subgroup of $G$, where the finite subset $D \subset X$ is the parabolic divisor, if and only if the $\mathscr{G}$-torsor is polystable.
\end{abstract}

\section{CONTEnTs}

1. Introduction

1.1. Origins

2. Preliminaries 4

2.1. Apartment of $G+5$

3. Parahoric group scheme and torsors

3.1. Invariant direct image

3.2. Parahoric torsors 6

3.3. Rational weights $\quad 7$

3.4. Rational weights and parahoric $\mathscr{G}$-torsors as $(\Gamma, G)$-bundles 8

4. Change of weights under a homomorphism 9

4.1. The local homomorphism problem 9

4.2. Facets of a homomorphism

5. Associated Constructions 11

5.1. The construction 11

5.2. Tensor product of parabolic vector bundles 11

5.3. Lie algebra bundle of a $\mathscr{G}$-torsor

6. Semistability and stability of torsors

6.1. First definition 12

6.2. Second definition

7. (Semi)stability and polystability under variation of weights 14

7.1. Facets of a quasi-parahoric torsor 14

8. Connections on parahoric $\mathscr{G}$-torsors $\quad 17$

2010 Mathematics Subject Classification. 14F22, 14D23, 14D20.

Key words and phrases. Bruhat-Tits group scheme; parahoric torsor; connection; polystability. 
8.1. $D_{X}$-modules 17

8.2. Logarithmic connections on curves

8.3. Connection on parabolic vector bundles 18

8.4. Lie connection on a principal $G$-bundle 18

8.5. Connection on parahoric $\mathscr{G}$-torsors

8.6. A Tannakian description of connection 19

9. Connections on $(\Gamma, G)$-bundles and rational weights 20

10. Flat unitary connections on parahoric torsors and stability 22

10.1. Polystable parahoric torsors 22

10.2. Polystable parahoric torsors from unitary representations 23

10.3. Polystable parahoric torsors and unitary representations 25

10.4. The reductive case 26

Acknowledgements

References

\section{INTRODUCTION}

Let $X$ be an irreducible smooth projective curve defined over the field of complex numbers. Let $G$ be a simple and simply connected affine algebraic group defined over $\mathbb{C}$. Fix a finite subset $D \subset X$. Let $\mathscr{G}$ be a Bruhat-Tits group scheme with parabolic points at $D$ (see Definition 3.3).

In $[\mathrm{BS}]$, an analogue of the Mehta-Seshadri theorem in [MS] was proved relating stable parahoric torsors under Bruhat-Tits group schemes with irreducible homomorphisms of certain Fuchsian groups into a maximal compact subgroup of $G$. This was done under the assumption that the parabolic weights are rational, or equivalently, the fixed points of the Fuchsian group are all elliptic. Recall that in $[\mathrm{BS}]$ it is shown that if the weights are chosen rational then one can recover the $\mathscr{G}$-torsors as invariant direct images of orbifold principal bundles with respect to suitable ramified covers of $X$ ramified over the parabolic points.

An obstruction to cover real weights in the setting of parahoric torsors is that the classical Bruhat-Tits group scheme is defined on spectra of discrete valuation rings while the phenomenon of parabolic bundles with real weights naturally lies in the setting of an analytic neighborhood of the origin. Further, the notion of invariant direct image fails to generalize directly to the analytic setting when the covering map is no longer algebraic. We address this issue by working with a natural analogue of Deligne extensions to the parahoric torsor setting; to a pair $(E, \widehat{d})$ of a principal $G$-bundle $E$ equipped with a flat connection $\widehat{d}$ on the punctured disc, we give a canonical extension to a torsor under Bruhat-Tits group scheme on the compact Riemann surface (see Section 10.2). We use this to construct the parahoric torsor associated to a representation of the fundamental group of the punctured Riemann surface. 
Another obstruction in case of real weights is the notion of stability for parahoric torsors. Filling the lacuna in [BS], we have a definition of stability for parahoric torsors (6.2) which covers real weights as well. Then, following the approach in the paper of MehtaSeshadri, [MS, p. 217], in Section 7 we develop the theme of variation of weights in the setting of parahoric torsors, where the notions of (semi)stability for the case of real weights are shown to coincide for "nearby" rational weights. Although the broad lines are the same this generalization is not entirely straight-forward.

We then go on to define the notion of a connection on a $\mathscr{G}$-torsor over a smooth projective curve over $\mathbb{C}$ and prove the analogue of the Donaldson-Uhlenbeck-Yau correspondence in the parahoric setting when the weights are real. The basic idea is to use the Tannakian formalism and the argument reduces to the case of the associated parabolic Lie algebra bundle. The more general reductive group case is then an easy generalization.

1.1. Origins. In the early 60s, Mumford defined the notion of stability for vector bundles on curves as a tool to get Hausdorff moduli spaces; using geometric invariant theory, he then constructed the moduli space of stable vector bundles. In ([NS|) Narasimhan and Seshadri gave an alternative characterization of stability using flat connections; more precisely, they proved that a holomorphic vector bundle $E$ on a compact Riemann surface is stable if and only if $E$ arises from an irreducible projective unitary representations of the fundamental group of the Riemann surface. This correspondence between flat projective unitary connections and stable vector bundles has been generalized in several directions. A. Ramanathan ( $[\mathrm{Ra} \mid)$ extended the correspondence to the case of holomorphic principal $G$-bundles, where $G$ is a complex reductive affine algebraic group [Ra| On the other hand, Mehta and Seshadri (|MS|) generalized the Narasimhan-Seshadri construction to include unitary logarithmic connections, or equivalently, to classify irreducible unitary representations of general Fuchsian groups with fixed conjugacy classes. These logarithmic connections are those which have regular singularity at finitely many points and were already apparent in the early work of Weil (|We $\mid$ ); their importance was emphasized by Deligne in $([\mathrm{De} \mid)$. The objects replacing stable bundles in the Mehta-Seshadri correspondence are stable parabolic vector bundles. Following Donaldson's reinterpretation of the Narasimhan-Seshadri correspondence, Biquard ( $|\overline{B i q}|)$ gave a differential geometric interpretation of this Mehta-Seshadri correspondence.

It is a very natural problem to generalize the Mehta-Seshadri correspondence from the setting of parabolic vector bundles to that of principal $G$-bundles, where $G$ is a complex reductive affine algebraic group. On the side of representations, the objects were easy to define; they were homomorphisms of Fuchsian groups taking values in a maximal compact subgroup of $G$ such that for each puncture of the Riemann surface the associated conjugacy class in the fundamental group of the surface is mapped to a fixed conjugacy class of the maximal compact subgroup of $G$. Similarly, on the side of connections, the corresponding objects were quite well-understood since the work of Deligne. The central problem was to generalize the notion of a stable parabolic vector bundle to the setting of principal $G$-bundles. From a Tannakian perspective ([BBN1]) it became apparent that a naive generalization in terms parabolic G-bundles, i.e., principal $G$ bundles with parabolic structures, was insufficient for setting up a comprehensive analogue of the Mehta-Seshadri correspondence. 
At a technical level, it was not clear what the correct notion of weight should be in the general setting. A breakthrough came in the work of Boalch $([\overline{\mathrm{Bo}} \mid)$ and Balaji-Seshadri $([\overline{\mathrm{BS}}])$. These papers introduced the correct and very natural notion of weight, namely, as a point in the affine apartment of $G$. As a consequence of this point view, the reason for the inadequacy of parabolic $G$-bundles also became clear. It was realized that instead of parabolic $G$-bundles one should consider torsors or principal homogeneous spaces under parahoric group schemes in the sense of Bruhat and Tits. Balaji and Seshadri $([\mathrm{BS} \mid)$ extended the Mehta-Seshadri theorem to the case of parahoric torsors with rational weights.

Prior to these works, there were at least two partial approaches to generalize the MehtaSeshadri theorem, both around the turn of the millennium. The approach in ([BBN1]) was Tannakian in spirit and followed the method of Nori [No]; this Tannakian approach identified the basic problem, namely that the object associated to a representation of a Fuchsian group into the maximal compact of $G$, such that for each puncture of the Riemann surface the associated conjugacy class in the fundamental group of the surface is mapped to a fixed conjugacy class of the maximal compact subgroup of $G$, in general will not be a principal $G$-bundle on the Riemann surface. The approach in ([TW]) again gave a partial solution to the problem; in the language of Weyl alcoves, the solution was for weights in the interior of the Weyl alcove which corresponds to the subclass of parabolic G-bundles.

Parahoric group schemes $\mathscr{G}$ and $\mathscr{G}$-torsors over smooth projective curves were first defined and studied by Pappas-Rapoport [PR1]. Subsequently, in [PR2], they made several conjectures on the moduli stacks of $\mathscr{G}$-torsors. Most of these conjectures were answered by Heinloth $([|\mathrm{He}|])$ providing a precise setting for the study of these moduli stacks. The paper of Seshadri ([كe2]), takes up the question of the analogue of the Mehta-Seshadri theorem; the main emphasis in ([Se2]) was again was to point out that for a solution to the problem of obtaining analogues of the Mehta-Seshadri theorem, one has to go beyond the category of principal $G$-bundles. Evidence to the role of Bruhat-Tits theory was also given in a few illustrative examples. The paper of Boalch ([Bo $]$ ) studies logarithmic connections on $G$-bundles and the notion of parahoric torsors comes along with the first appearance of the notion of weights for these torsors. Around the same time and independently in [BS], a similar notion of weights was defined towards providing a satisfactory analogue of the Mehta-Seshadri theorem in the general setting for semisimple groups $G$, thereby completing the broad picture outlined in [Se2]. The notion of "invariant direct images" of torsors in [BS] plays a key role analogous to the one in [MS] for the case of vector bundles.

\section{Preliminaries}

In this section we collect together some standard notions and notation that will be used throughout this paper. See [BT1], [BT2], [BS], [He1] for this section and the next one.

The base field will always be $\mathbb{C}$.

Define

$$
A:=\mathbb{C}[[t]] \text { and } K:=\mathbb{C}((t)),
$$

where $t$ denote a uniformizing parameter. Let $G$ be a semisimple simply connected affine algebraic group defined over $\mathbb{C}$. The Lie algebra of $G$ will be denoted by $\mathfrak{g}$. Fix a maximal torus $T \subset G$; let $Y(T)=\operatorname{Hom}\left(\mathbb{G}_{m}, T\right)$ be the group of all holomorphic one-parameter subgroups of $T$. 
2.1. Apartment of $G$. For each maximal torus $T$ of $G$, we have the standard affine apartment $\mathscr{A}_{T}$. It is an affine space under $Y(T) \otimes_{\mathbb{Z}} \mathbb{R}$. In general, there is no origin in the apartment (cf. [BT1]). But for purposes of this paper, we shall identify $\mathscr{A}_{T}$ with $Y(T) \otimes_{\mathbb{Z}} \mathbb{R}$ (see [BS, § 2]).

Let $\mathcal{V}$ be a real affine space. A function $f: \mathcal{V} \longrightarrow \mathbb{R}$ is said to be an affine functional if

$$
f(r x+(1-r) y)=r f(x)+(1-r) f(y)
$$

for all $x, y \in \mathcal{V}$ and $r \in \mathbb{R}$. Thus, for a root $\alpha$ of $G$ and an integer $n \in \mathbb{Z}$ there is the affine functional

$$
\alpha+n: \mathscr{A}_{T} \longrightarrow \mathbb{R}, x \longmapsto \alpha(x-0)+n .
$$

We note that these are called the affine roots of $G$. The zero locus of $\alpha+n$ will be denoted by $H_{\alpha+n}$; it is called an affine wall. The set of affine walls is known to be locally finite, meaning any compact subset of $\mathscr{A}_{T}$ intersects only finitely many affine walls. For any point $x \in \mathscr{A}_{T}$, let $Z_{x}$ denote the set of affine functionals vanishing on $x$. For an integer $n \geq 0$, define

$$
\mathscr{H}_{n}=\left\{x \in \mathscr{A}|\quad| Z_{x} \mid=n\right\},
$$

which is the set of all points where $n$ of the affine functionals vanish. A facet $\Omega$ of $\mathscr{A}_{T}$ is defined to be a connected component of $\mathscr{H}_{n}$ for some $n$. The dimension of a facet is its dimension as a real manifold. We then have a decomposition of the apartment

$$
\mathscr{A}_{T}=\bigsqcup_{n} \mathscr{H}_{n} \text {. }
$$

Although, as mentioned above, almost always $\Theta$ will be a point of $\mathscr{A}_{T}$, sometimes $\Theta$ will also be a facet of $\mathscr{A}_{T}$.

\section{PARAHORIC GROUP SCHEME AND TORSORS}

3.1. Invariant direct image. The base field is $\mathbb{C}$. Let $p: W \longrightarrow U$ be a finite flat surjective morphism of normal, integral Noetherian schemes which is Galois. So the Galois group $\operatorname{Gal}(p)$, which we will denote by $\Gamma$, acts on $W$ with $U=W / \Gamma$ being the quotient. Such a morphism $p$ is called a Galois covering with Galois group $\Gamma$.

Let $\mathscr{G}$ be an affine group scheme over $W$. For the above Galois covering map $p$, the direct image $p_{*} \mathscr{G}$ is defined as follows: for each $U$-scheme $S$, set

$$
\left(p_{*} \mathscr{G}\right)(S)=\operatorname{Hom}_{W}\left(S \times_{U} W, \mathscr{G}\right) ;
$$

this is representable by a group scheme [BLR, Theorem 4 and Proposition 6]. Assume that the group scheme $\mathscr{G}$ is equipped with an action of the Galois group $\Gamma$ that lifts the action of $\Gamma$ on $W$; in particular, the "multiplication map" and the "inverse map" on $\mathscr{G}$ are $\Gamma$-equivariant. Such a $\mathscr{G}$ will be called a $\Gamma$-group scheme over $W$.

There is a left action of $\Gamma$ on $S \times{ }_{U} W$ induced by the action of $\Gamma$ on $W$. This and the left action of $\Gamma$ on $\mathscr{G}$ together induce the following right action of $\Gamma$ on $\left(p_{*} \mathscr{G}\right)(S)$ :

$$
(f \cdot \gamma)([s, w]):=\gamma^{-1} \cdot f(\gamma \cdot[s, w]), \quad[s, w] \in S \times_{U} W, \gamma \in \Gamma .
$$

Consider the fixed point subscheme under the above action of $\Gamma$ on $p_{*} \mathscr{G}$. The general results on fixed point subschemes given in [Ed, Section 3] can be applied to our situation 
since the characteristic is 0 . Consequently, a canonically defined smooth closed $X$-subgroup scheme

$$
p_{*}^{\Gamma} \mathscr{G}:=\left(p_{*} \mathscr{G}\right)^{\Gamma} \subset p_{*} \mathscr{G}
$$

is obtained. This $p_{*}^{\Gamma} \mathscr{G}$ is representable because $p_{*} \mathscr{G}$ is representable.

Definition 3.1 (Invariant direct image). Let $p: W \longrightarrow U$ be as above, and let $\Gamma=\operatorname{Gal}(W / U)$. Let $\mathscr{G}$ be a smooth affine $\Gamma$-group scheme over $W$. Define the invariant direct image of $\mathscr{G}$ to be

so $\left(p_{*}^{\Gamma} \mathscr{G}\right)(S)=\mathscr{G}\left(S \times_{U} W\right)^{\Gamma}$ for any $U$-scheme $S$.

$$
p_{*}^{\Gamma}(\mathscr{G}):=\left(p_{*} \mathscr{G}\right)^{\Gamma},
$$

More generally, if $E$ is any affine scheme over $W$ with a lift of the $\Gamma$-action on $W$, then define the invariant direct image of $E$ to be

$$
p_{*}^{\Gamma} E:=\left(p_{*} E\right)^{\Gamma} .
$$

3.2. Parahoric torsors. Notation of Section 2.1 will be followed. Let $R=R(T, G)$ denote the root system of $G$ (cf. [Sp, p. 125]). Thus for every $r \in R$, there is the root homomorphism $u_{r}: \mathbb{G}_{a} \longrightarrow G[\mathrm{Sp}$, Proposition 8.1.1].

For any non-empty subset $\Theta \subset \mathscr{A}_{T}$, the parahoric subgroup $\mathscr{P}_{\Theta} \subset G(K)$

$$
\mathscr{P}_{\Theta}:=\left\langle T(A),\left\{u_{r}\left(t^{m_{r}(\Theta)} A\right)\right\}_{r \in R}\right\rangle .
$$

is the subgroup generated by $T(A)$ and $\left\{u_{r}\left(t^{m_{r}(\Theta)} A\right)\right\}_{r \in R}$, where

$$
m_{r}=m_{r}(\Theta)=-\left[\inf _{\theta \in \Theta}(\theta, r)\right],
$$

and $A$ is defined in (2.1) [BS, Page 8]. Moreover, by [BT2, Section 1.7] we have an affine flat smooth group scheme $\mathscr{G}_{\Theta} \longrightarrow \operatorname{Spec}(A)$ corresponding to $\Theta$. The set of $K$-valued (respectively, $A$-valued) points of $\mathscr{G}_{\Theta}$ is identified with $G(K)$ (respectively, $\mathscr{P}_{\Theta}$ ). The group scheme $\mathscr{G}_{\Theta}$ is uniquely determined by its $A$-valued points. Here we will often take $\Theta$ to be just a point of $\mathscr{A}_{T}$.

Remark 3.2. We remark that the notion of a parahoric subgroup is defined in the greatest generality in the basic papers of Bruhat and Tits. For our purposes, the definition given above is sufficient.

Let $X$ be a smooth complex projective curve. We fix once for all a nonempty finite set of closed points

$$
D=\left\{x_{j}\right\}_{j=1}^{m} \subset X .
$$

These will play the role of parabolic points. Let $A_{j}:=\widehat{\widetilde{O}_{X, x_{j}}}$ be the complete discrete valuation ring with function field $K_{j} \simeq \mathbb{C}((t))$ and residue field $\mathbb{C}$, obtained by completing the local rings $\mathscr{O}_{X, x_{j}}$. We shall denote $\operatorname{Spec}\left(A_{j}\right)$ by $D_{j}$.

Definition 3.3. Let $\mathscr{G}$ be a flat, affine group scheme on $X$ of finite type. We call $\mathscr{G}$ a BruhatTits group scheme with parabolic points $D$, if

(1) restricted to $X \backslash D$, it is isomorphic to the split group scheme $G \times(X \backslash D)$, and

(2) $\left.\mathscr{G}\right|_{D_{j}} \longrightarrow D_{j}$ is a Bruhat-Tits group scheme for each $j$.

We shall denote $\mathscr{G}$ by $\mathscr{G}_{\Omega}$, where $\Omega=\left\{\Omega_{j}\right\}_{j=1}^{m}$ is a collection of facets of the Bruhat-Tits building with $\mathscr{G}_{D_{j}}$ corresponding to $\Omega_{j}$. 
By the general theory due to Bruhat and Tits, one has an affine flat smooth group scheme $\mathscr{G}_{\Theta}$ on $\operatorname{Spec}(A)$ corresponding to $\Theta$. The set of $K$-valued (respectively, $A$-valued) points of $\mathscr{G}_{\Theta}$ is identified with $G(K)$ (respectively, $\mathscr{P}_{\Theta}$ ). The group scheme $\mathscr{G}_{\Theta}$ is uniquely determined by its $A$-valued points. These notions were defined and the moduli stack of $\mathscr{G}_{\Theta}-$ torsors studied extensively in the papers of Pappas-Rapoport (cf. [PR1], [TR2]) and Heinloth $(\mid[\mathrm{He}||)$. To construct one on the whole of $X$ one can proceed as in [BS, 5.2]. Existence of such group schemes also follows from the invariant direct images constructed above (see also [BS, Theorem 5.2.7]).

Definition 3.4 ([BSS, Section 6]). A quasi-parahoric torsor $\mathscr{E}$ is a $\mathscr{G}_{\Omega, X}$-torsor on $X$.

Definition 3.5 ([B], Section 6$]$, $[\overline{\mathrm{BO}}])$. A parahoric torsor is a pair $(\mathscr{E}, \boldsymbol{\theta})$ consisting of

(1) a $\mathscr{G}_{\Omega, X}$-torsor $\mathscr{E} \longrightarrow X$, and

(2) weights, meaning elements $\boldsymbol{\theta}=\left\{\theta_{i}\right\}_{i=1}^{m} \in(Y(T) \otimes \mathbb{R})^{m}$ in the interior of $\Omega_{i}$.

Remark 3.6. The above notion of weight is the precise analogue of the classical weight for a parabolic vector bundle with multiplicity (cf. [MS, page 211, Definition 1.5]).

Remark 3.7. It should be noted that as in $[\overline{\mathrm{BS}}]$, the theory of Bruhat-Tits group schemes that are used here assumes that the group $G$ is semisimple and simply connected. On the other hand, for the case of GL( $V)$, which satisfies neither of these conditions, the parabolic bundles is classical (cf. [Se1], [MS], [Bis]). In [BS, Example 2.3.4] and [BS, Remark 6.1.5], it is noted that the torsors under Bruhat-Tits group schemes for GL $(V)$ are same as the parabolic vector bundles. Let us spell this out for the convenience of the reader. Let $\mathscr{G} \mathscr{L}(V)$ be a Bruhat-Tits group scheme on $\operatorname{Spec}(A)$ with generic fiber $\operatorname{GL}(V)$. Fix a maximal torus $T(V) \subset \mathrm{GL}(V)$. Let $\mathscr{E}$ be a $\mathscr{G} \mathscr{L}(V)$-torsor, and let $\theta(V) \in A l(T(V))_{\mathbb{R}}$ be a weight as a point in the so-called Weyl alcove (see [BS, p. 9]). Then, the associated vector bundle gets a canonical parabolic structure with quasi-parabolic type determined by the group scheme $\mathscr{G} \mathscr{L}(V)$ while the parabolic weights are given by $\theta(V)$. We observe that in the case of GL $(n, \mathbb{C})$, or $\operatorname{SL}(n, \mathbb{C})$, giving a point $\theta(V) \in A l(T(V))_{\mathbb{R}}$ in the Weyl alcove is equivalent to giving $n$-tuples $\left(\alpha_{1}, \alpha_{2}, \cdots, \alpha_{n}\right) \in \mathbb{R}^{n}$, such that every $\alpha_{i} \geq 0$ and $\alpha_{i} \leq \alpha_{i+1}$, for $1 \leq i \leq n-1$; in the case of $\operatorname{SL}(n, \mathbb{C})$, the condition of the determinant translates to the further condition

$$
\sum_{i=1}^{n} \alpha_{i} \in \mathbb{Z}
$$

By a Weyl group conjugation, we can also arrange the $\alpha_{i}$ 's in increasing order, i.e., $0 \leq \alpha_{1} \leq$ $\alpha_{2} \leq \cdots \leq \alpha_{n}<1$. Once the parahoric subgroup is chosen, this determines a flag type and hence we may even order the parabolic weights in a strictly increasing sequence.

3.3. Rational weights. Starting with a $m$-tuple of weights $\boldsymbol{\theta} \in(Y(T) \otimes \mathbb{Q})^{m}$, following the proof of the converse in [BS, Theorem 2.3.1], we get positive integers $d_{1}, d_{2}, \cdots, d_{m}$ such that $d_{i} \cdot \theta_{i} \in Y(T)$. By choosing these $d_{i}$ to be smallest with this property, we see that a choice of $\boldsymbol{\theta}$ entails a choice of ramification index $d_{x}$ at each point $x \in D$.

There exists a ramified Galois cover of curves $p: Y \longrightarrow X$ which is

- unramified over $X \backslash D$, and

- the ramification index over $x \in D$ is $d_{x}$,

if and only if exactly one of the following conditions hold: 
(1) the genus of $X$ is nonzero,

(2) $X=\mathbb{P}^{1}$ and $\# D \geq 3$,

(3) $X=\mathbb{P}^{1}, \# D=2$, and $d_{x}=d_{y}$, where $D=\{x, y\}$.

(See [Na, p. 26, Proposition 1.2.12].)

When we consider parahoric torsors as invariant direct images of $(\Gamma, G)$ bundles, it will always be with respect to such a Galois cover. See also [Bis].

3.4. Rational weights and parahoric $\mathscr{G}$-torsors as $(\Gamma, G)$-bundles. Let $(X, D)$ be as above, and let $\mathscr{G} \longrightarrow X$ be a Bruhat-Tits group scheme over $X$. By [BS, Theorem 5.3.1], there exists a (possibly ramified) finite Galois cover $p: Y \longrightarrow X$ branched over $D$, and a principal $G-$ bundle $F$ over $Y$ (cf. [BS, Notation 5.1.0.1]) equipped with a lift of the action of the Galois $\operatorname{group} \Gamma:=\operatorname{Gal}(p)$, such that the following statements hold:

(1) Let ${ }_{F} G:=\operatorname{Isom}_{Y}(F, F)$ be the twisting of the constant group scheme $G \longrightarrow Y$ by $F$. The invariant direct image satisfies the condition

$$
p_{* F}^{\Gamma} G=\mathscr{G} .
$$

(2) Let $D_{Y}:=p^{-1}(D) \subset Y$ denote the ramification points of the covering $p$. For each $y \in D_{Y}$, let $\Gamma_{y} \subset \Gamma$ be the isotropy subgroup that fixes $y$. Let $\tau_{y}: \Gamma_{y} \longrightarrow \operatorname{Aut}\left(F_{y}\right)$ be the action of $\Gamma_{y}$ on the fiber $F_{y}$. Its conjugacy class is called the local type of $F$ at $y$; this local type will be denoted by $\left[\tau_{y}\right]$. By the type $\tau$ of $F$, we shall mean the set of conjugacy classes $\left[\tau_{y}\right]$ of $\tau_{y}$ :

$$
\tau:=\left\{\left[\tau_{y}\right] \mid y \in D_{Y}\right\} .
$$

Let $\mathscr{M}_{Y}^{\tau}(\Gamma, G)$ denote the moduli stack of $(\Gamma, G)$ bundles over $Y$ of type $\tau$, and let $\mathscr{M}_{X}(\mathscr{G})$ denote the moduli stack of $\mathscr{G}$ torsors on $X$. Then there is an isomorphism of moduli stacks

$$
\alpha_{F}: \mathscr{M}_{Y}^{\tau}(\Gamma, G) \longrightarrow \mathscr{M}_{X}(\mathscr{G}),
$$

given by the $(\Gamma, G)$ bundle $F$ as follows: Denote by $F^{o p}$ the left $G$-bundle defined by $g f:=f g^{-1}$, where $g \in G$ and $f \in F$. The above group scheme ${ }_{F} G$ acts on the right of $F^{o p}$. The isomorphism in (3.7) is:

$$
E \longmapsto p_{*}^{\Gamma}\left(E \times_{Y, G} F^{o p}\right)=p_{*}^{\Gamma}\left(\operatorname{Isom}_{Y}(E, F)\right) .
$$

The inverse of the map in (3.7) is given by

$$
\mathscr{E} \longmapsto p^{*} \mathscr{E} \times p^{* \mathscr{G}} F .
$$

(3) Let $y \in D_{Y}$, and $x:=p(y)$. Let $N_{y}=\operatorname{Spec}(B)$, where $B=\widehat{\mathscr{O}_{Y, y}}$, and also $D_{x}=$ $\operatorname{Spec}(A)$ with $A=\widehat{\mathscr{O}_{X, x}}$. Let $U_{y}$ denote the group of local $\Gamma_{y}-G$ automorphisms of $\left.F\right|_{N_{y}}$ (cf. [BS, Definition 2.2.7]). Then by [BS, Proposition 5.1.2] we have

$$
\mathscr{G}_{D_{x}}(A)=U_{y} .
$$

For the equality in (3.10), we need the existence of $(\Gamma, G)$ bundle $F$ only locally on $N_{y}$ and not on entire $Y$.

A different $(\Gamma, G)$ bundle $F^{\prime}$ of type $\tau$ will, in general, provide a different (cf. (3.7)) isomorphism $\alpha_{F^{\prime}}: \mathscr{M}_{Y}^{\tau}(\Gamma, G) \longrightarrow \mathcal{M}_{X}(\mathscr{G})$. 


\section{CHANGE OF WeightS UNDER A HOMOMORPHISM}

4.1. The local homomorphism problem. Consider the following problem. Let $A$ be an arbitrary discrete valuation ring with quotient field $K$. Let $G$ be semisimple and simply connected group. Let $\rho: G \longrightarrow H$ be a homomorphism. Fix a maximal torus $T \subset G$. Then fix a weight $\theta$ in the affine apartment $\mathscr{A}_{T}$ for $T$. It should be emphasized that $\theta$ may not be a rational point, meaning it may not lie in the image of $Y(T) \otimes_{\mathbb{Z}} \mathbb{Q}$. Fix a maximal torus $T_{H} \subset H$ such that $\rho(T) \subset T_{H}$. Via the identification between $\mathscr{A}_{T}$ and $Y(T) \otimes_{\mathbb{Z}} \mathbb{R}$ mentioned in Section 2.1, we have a linear map

$$
\mathscr{A}_{T} \longrightarrow \mathscr{A}_{T_{H}}
$$

between the apartments. Let $\theta_{H}$ denote the image of $\theta$ under this map. We wish to construct, through $\rho$, a canonical homomorphism of group schemes over $\operatorname{Spec}(A)$ : from $\mathscr{G}_{\theta}$ corresponding to $\theta$ to $\mathscr{G}_{\theta_{H}}$ corresponding to $\theta_{H}$.

4.2. Facets of a homomorphism. Let $\rho: G \longrightarrow H$ be a homomorphism. The affine roots of $H$ give affine functionals on $\mathscr{A}_{T_{H}}$ (cf. Section 2). These functionals are defined over the rationals. By (4.1) it follows that the linear map of apartments $\mathscr{A}_{T} \longrightarrow \mathscr{A}_{T_{H}}$ corresponding to $\rho$ is also defined over the rationals. Indeed, it is induced by the algebraic map $\left.\rho\right|_{T}: T \longrightarrow$ $T_{H}$. Via $\mathscr{A}_{T} \longrightarrow \mathscr{A}_{T_{H}}$, we view the above affine functionals on $\mathscr{A}_{T_{H}}$, associated to the affine roots of $H$, as affine functionals on $\mathscr{A}_{T}$. Take the union of these affine functionals with the usual affine functionals on $\mathscr{A}_{T}$ corresponding to the affine roots of $G$. Note that all these functionals are defined over rationals. We shall call them as $\rho$-functionals.

Definition 4.1. An affine wall of $\rho$ is the zero locus of a $\rho$-functional. For any $x \in \mathscr{A}_{T}$, define $Z_{x}$ to be the set of all affine functionals on $\mathscr{A}_{T}$ vanishing at $x$. Define

$$
\mathscr{H}_{n}:=\left\{x \in \mathscr{A}|\quad| Z_{x} \mid=n\right\} .
$$

A facet of $\rho$ is a connected component of $\mathscr{H}_{n}$ for some $n \geq 0$.

Notice that for the identity homomorphism $G \longrightarrow G$, we have just got the usual facets. By the theory of buildings, one knows that the usual affine walls of $G$ provide a decomposition of $\mathscr{A}_{T}$ (cf. (2.2)).

We claim that more generally, the facets of a homomorphism $\rho: G \longrightarrow H$ provide an even finer decomposition of

$$
\mathscr{A}_{T}=\bigsqcup_{n} \mathscr{H}_{n}
$$

here $n$ varies over a finite set. To prove this claim, first note that the set of all $\rho$-affine walls corresponding to $\rho$-functionals form a locally finite set. Indeed, this follows because any compact set $C$ of $\mathscr{A}_{T}$ meets finitely many usual affine walls in $\mathscr{A}_{T}$. Now under the map $\mathscr{A}_{T} \longrightarrow \mathscr{A}_{T_{H}}$, the image of $C$ being compact, meets finitely many affine walls of $\mathscr{A}_{T_{H}}$. Thus $C$ meets only finitely many walls of $\rho$.

Proposition 4.2. Let $\rho: G \longrightarrow H$ be a homomorphism of algebraic groups. Given a weight $\theta \in \mathscr{A}_{T}$, there exists a rational weight $\eta \in \mathscr{A}_{T}$ lying in the same facet as $\theta$ such that $\eta_{H}$ and $\theta_{H}$ also lie in a common facet of $\mathscr{A}_{T_{H}}$.

Proof. It can be shown that if the element $\theta \in \mathscr{A}_{T}$ lies in a zero dimensional facet, then it must be a rational point. Indeed, this follows from that fact that we are looking at common 
zero locus of a set of rational functionals. Taking contrapositive of the last statement, if $\theta$ is not rational then a $\rho$-facet of $\theta$ cannot be zero dimensional. So we can find a rational weight $\eta$ in it.

Now by construction, both $\eta$ and $\theta$ lie in the same facet of $\mathscr{A}_{T}$, and also $\theta_{H}$ and $\eta_{H}$ lie in a common facet of $\mathscr{A}_{T_{H}}$.

Remark 4.3. Henceforth, when we say that a rational weight $\eta$ is close to $\theta$ with respect to $\rho: G \longrightarrow H$, we mean a rational weight in the sense of Proposition 4.2 .

The constructions in the proof of Proposition 4.2 can be extended to the context of a finite set of representations $\left\{\rho_{i}: G \longrightarrow \mathrm{GL}\left(V_{i}\right)\right\}_{i \leq n}$ of $G$.

Now further assume that $\eta$ and $\theta$ are actually interior points of one $\rho$-facet. This assumption implies that they define isomorphic Bruhat-Tits group schemes with generic fiber $G$ and also group schemes with generic fiber $H$. There are canonical homomorphisms between them by the following proposition.

Proposition 4.4. Let $\rho: G \longrightarrow H$ be given. Let $\theta \in \mathscr{A}_{T}$ be a weight with image $\theta_{H}$ under the map of apartments $\mathscr{A}_{T} \longrightarrow \mathscr{A}_{T_{H}}$. Then there is a canonical homomorphism of group schemes $\mathscr{G}_{\theta} \longrightarrow \mathscr{G}_{\theta_{H}}$ over $\operatorname{Spec}(A)$.

Proof. Let us recall a characterization of parahoric groups when the weight $\eta$ lies in $\mathscr{A}_{\mathbb{Q}}$ Theorem 2.3.1]. In this case, by (3.10), there exists a ramified Galois cover

$$
p: \operatorname{Spec}(B) \longrightarrow \operatorname{Spec}(A)
$$

with Galois group say $\Gamma$, and a $(\Gamma, G)$ bundle $F \longrightarrow \operatorname{Spec}(B)$, such that

$$
\mathscr{G}_{\Omega(A)}=\mathscr{G}_{\eta}(A)=\operatorname{Aut}_{(\Gamma, G)}(F) .
$$

Choose a rational weight $\eta$ close to $\theta$ with respect to $\rho$. This gives an element $\eta_{H}$ close to $\theta_{H}$. Further, let

$$
F(H):=F \times{ }_{G} H
$$

be the $(\Gamma, H)$ bundle obtained by extending structure group using $\rho$. Via the natural homomorphism $\operatorname{Aut}_{(\Gamma, G)}(F) \longrightarrow \operatorname{Aut}_{(\Gamma, H)}(F(H))$ and the equalities $\mathscr{G}_{\eta}(A)=\operatorname{Aut}_{(\Gamma, G)}(F)$ and $\mathscr{G}_{\eta_{H}}(A)=\operatorname{Aut}_{(\Gamma, H)}(F(H))$, we obtain a map of parahoric groups

$$
\rho_{K}: \mathscr{G}_{\eta}(A) \longrightarrow \mathscr{G}_{\eta_{H}}(A) \text {. }
$$

Now the characterizing property of the Bruhat-Tits group schemes is that they are étoffé, which means that any morphism at the level of group schemes over $\operatorname{Spec}(A)$ is determined completely by the $A$-valued points alone (cf. [BT2, Definition 1.7.1]).

Thus we get a morphism over $\operatorname{Spec}(A)$ of group schemes

$$
\mathscr{G}_{\eta} \longrightarrow \mathscr{G}_{\eta_{H}}
$$

extending (4.2). Since $\eta_{H}$ is close to $\theta_{H}$, it follows that $\mathscr{G}_{\eta_{H}}(A)=\mathscr{G}_{\theta_{H}}(A)$, and hence we have an induced isomorphism of group schemes $\mathscr{G}_{\eta_{H}} \longrightarrow \mathscr{G}_{\theta_{H}}$ on $\operatorname{Spec}(A)$ which gives the homomorphism $\rho_{\theta}: \mathscr{G}_{\theta} \longrightarrow \mathscr{G}_{\theta_{H}}$.

To the best of our knowledge, Proposition 4.4 is not available in the papers of Bruhat and Tits. Alternatively it can be proved using the general framework of functoriality of buildings as in [La, Theorem 2.1.8] and [La, Theorem 2.2.1]. 


\section{Associated Constructions}

As before, $G$ is semisimple and simply connected. Let $\rho: G \longrightarrow \operatorname{GL}(V)$ be a rational representation. Fix a maximal torus $T \subset G$, and also fix a maximal torus $T_{V} \subset \mathrm{GL}(V)$ containing $\rho(T)$. Let $\mathscr{E}$ be a parahoric $\mathscr{G}_{\boldsymbol{\theta}, X}$-torsor on $X$ with weights $\boldsymbol{\theta}$.

5.1. The construction. As in (3.5), fix a finite subset $D=\left\{x_{j}\right\}_{j=1}^{m} \subset X$. For each parahoric group scheme $\mathscr{G}_{j} \longrightarrow D_{j}=\operatorname{Spec}\left(A_{j}\right), 1 \leq j \leq m$, we get a facet $\Omega_{j}$ in the Bruhat-Tits building $\mathscr{B}(G)$. Any weight $\boldsymbol{\theta} \in \mathscr{A}_{T}^{m}$ induces a weight $\boldsymbol{\theta}_{V}$ in $\mathscr{A}_{T(V)}^{m}$ (see Section 4.2 ).

The group scheme $\mathscr{G}_{\boldsymbol{\theta}, X}$ on $X$ is obtained by gluing $\mathscr{G}_{\theta_{j}}$, for every $1 \leq j \leq m$, with

$$
\left.\mathscr{G}\right|_{X \backslash D} \simeq(X \backslash D) \times G \text {. }
$$

Using the same gluing data via the representation $\rho$ one immediately gets a Bruhat-Tits group scheme $\mathscr{G} \mathscr{L}_{\theta_{V}, X}$, and using locally Proposition 4.4, we see that $\rho$ gives a natural global homomorphism of group schemes over $X$

$$
\rho_{X}: \mathscr{G}_{\boldsymbol{\theta}, X} \longrightarrow \mathscr{G}_{\theta_{V}, X}
$$

Using $\rho_{X}$ one gets the standard construction of extension of structure groups.

By Remark 3.7, via $\rho$ one obtains an associated parabolic vector bundle

$$
\mathscr{E}_{V, \boldsymbol{\theta}_{V}}:=\left(\mathscr{E}(V), \boldsymbol{\theta}_{V}\right)
$$

with parabolic weights $\boldsymbol{\theta}_{V}$. We will mostly need to apply associated constructions under the adjoint homomorphism $A d: G \longrightarrow \mathrm{GL}(\mathfrak{g})$.

5.2. Tensor product of parabolic vector bundles. Assume that we have homomorphisms $\rho_{1}: G \longrightarrow \mathrm{GL}(V)$ and $\rho_{2}: G \longrightarrow \mathrm{GL}(W)$, and let

$$
\rho_{1} \otimes \rho_{2}: G \longrightarrow \mathrm{GL}(V \otimes W)
$$

be their tensor product. Therefore, for a parahoric torsor $(\mathscr{E}, \theta)$ we get parabolic vector bundles $\mathscr{E}_{V, \boldsymbol{\theta}_{V}}, \mathscr{E}_{W, \boldsymbol{\theta}_{W}}$, and $\mathscr{E}_{V \otimes W, \boldsymbol{\theta}_{V \otimes W}}$.

When the weights $\boldsymbol{\theta}$ are rational numbers then, by $(\Gamma, G)$ bundle theory, we have a canonical isomorphism of parabolic vector bundles $[\mathrm{MY} \mid$ :

$$
\mathscr{E}_{V, \boldsymbol{\theta}_{V}} \otimes^{p} \mathscr{E}_{W, \boldsymbol{\theta}_{W}} \simeq \mathscr{E}_{V \otimes W, \boldsymbol{\theta}_{V \otimes W}},
$$

where $\otimes^{p}$ is parabolic tensor product. The following proposition, which extends this isomorphism to the case of real weights, is immediate by observing that the quasi-parabolic bundle for a parabolic tensor product does not change under sufficiently small change of the parabolic weights of the factors, while the parabolic weights of the parabolic tensor product are given by the parabolic weights of the factors using a standard algebraic formula.

Proposition 5.1. Let $\theta \in \mathscr{A}_{T}$ be arbitrary. Then there is a canonical isomorphism

$$
\mathscr{E}_{V, \boldsymbol{\theta}_{V}} \otimes^{p} \mathscr{E}_{W, \theta_{W}} \simeq \mathscr{E}_{V \otimes W, \theta_{V \otimes W}}
$$

of parabolic vector bundles. 
Let $V$ and $W$ be two representations of $G$ and $\psi: V \longrightarrow W$ a $G$-equivariant homomorphism. Let $(\mathscr{E}, \boldsymbol{\theta})$ be a parahoric torsor. By identifying the $G$-module $\operatorname{Hom}(V, W)$ with $W \otimes V^{*}$ it follows that $\psi$ induces a homomorphism

$$
(\mathscr{E}, \boldsymbol{\theta})(\psi): \mathscr{E}_{V, \boldsymbol{\theta}_{V}} \longrightarrow \mathscr{E}_{W, \boldsymbol{\theta}_{W}}
$$

5.3. Lie algebra bundle of a $\mathscr{G}$-torsor. Consider a parahoric $\mathscr{G}_{\boldsymbol{\theta}}$-torsor $(\mathscr{E}, \boldsymbol{\theta})$, where $\boldsymbol{\theta}$ is a system of real weights. We define a parabolic Lie bracket operation on $\mathscr{E}(\mathfrak{g})$ as follows. The Lie bracket [,] $: \mathfrak{g} \otimes \mathfrak{g} \longrightarrow \mathfrak{g}$ is a $G$-equivariant homomorphism for the adjoint action of $G$, and hence by (5.2) we have a homomorphism of parabolic vector bundles

$$
\mathscr{E}_{[,]}: \mathscr{E}(\mathfrak{g} \otimes \mathfrak{g}) \longrightarrow \mathscr{E}(\mathfrak{g}) .
$$

Now Proposition 5.1 gives an isomorphism

$$
\mathscr{E}(\mathfrak{g}) \otimes^{p} \mathscr{E}(\mathfrak{g}) \stackrel{\sim}{\longrightarrow} \mathscr{E}(\mathfrak{g} \otimes \mathfrak{g})
$$

of parabolic vector bundles. Combining with $\mathscr{E}_{[,]}$, the Lie bracket can be defined as the parabolic homomorphism

$$
[., .]: \mathscr{E}(\mathfrak{g}) \otimes_{X}^{p} \mathscr{E}(\mathfrak{g}) \longrightarrow \mathscr{E}(\mathfrak{g})
$$

\section{SEMISTABILITY AND STABILITY OF TORSORS}

Let $\mathscr{G}_{\Omega, X}$ be a Bruhat-Tits group scheme on $X$ as in Definition 3.3, and let $\boldsymbol{\theta}$ be such that $\mathscr{G}_{\Omega, X} \simeq \mathscr{G}_{\boldsymbol{\theta}, X}$. Let $(\mathscr{E}, \boldsymbol{\theta})$ be a parahoric $\mathscr{G}_{\boldsymbol{\theta}, X}$-torsor with arbitrary real weights $\boldsymbol{\theta} \in \mathscr{A}_{T}^{m}$.

Remark 6.1. Let $G_{K}$ be a split group scheme over a field $K$. Let $E_{K}$ be a $G_{K}$-torsor. Consider the twisted group scheme $E(G)_{K}$. Then giving a parabolic subgroup scheme

$$
P_{K} \subset E(G)_{K}
$$

(of fiber type $P$ ) is equivalent to giving a reduction of structure group of $E_{K}$ to $P$ (cf. [SGA, Exposé XXVI, Cor. 3.6]).

6.1. First definition. Let $\mathscr{E}(\mathscr{G})$ denote the group scheme of automorphisms of $\mathscr{E}$ obtained by taking the quotient $\mathscr{E} \times_{X} \mathscr{G}$ by the left $\mathscr{G}$-action on $\mathscr{E}$ and the right $\mathscr{G}$-action on itself by conjugation. Let $\operatorname{Lie}(\mathscr{E}(\mathscr{G}))$ denote the Lie algebra bundle of $\mathscr{E}(\mathscr{G})$. One has the following well-known identification of Lie algebra bundles:

$$
\operatorname{Lie}(\mathscr{E}(\mathscr{G}))=\mathscr{E}(\mathfrak{g}) .
$$

Since $(\mathscr{E}, \boldsymbol{\theta})$ is a parahoric torsor, by Section 5.3 , the above Lie algebra bundle $\mathscr{E}(\mathfrak{g})$ gets a natural parabolic Lie algebra bundle structure $\left(\mathscr{E}(\mathfrak{g}), \theta_{\mathfrak{g}}\right)$. Thus via the isomorphism (6.1) the bundle $\operatorname{Lie}(\mathscr{E}(\mathscr{G}))$ gets a parabolic vector bundle structure with a Lie bracket operation compatible with the parabolic structure:

$$
\left(\operatorname{Lie}(\mathscr{E}(\mathscr{G})), \theta_{\mathfrak{g}},[., .]\right)
$$

By Remark6.1, giving a generic parabolic reduction is equivalent to giving a parabolic subgroup scheme

$$
P_{K} \subset \mathscr{E}\left(\mathscr{G}_{K}\right) .
$$

For each $1 \leq j \leq m$, we take the flat closure of $P_{K}$ in $\mathscr{E}\left(\mathscr{G}_{A_{j}}\right)$. This will give a subgroup scheme $\mathscr{P} \subset \mathscr{E}(\mathscr{G})$. 
The extended subgroup scheme $\mathscr{P}$ also gives a Lie subalgebra bundle

$$
\operatorname{Lie}(\mathscr{P}) \subset \operatorname{Lie}(\mathscr{E}(\mathscr{G})) \simeq \mathscr{E}(\mathfrak{g}) .
$$

Then endow the bundle $\operatorname{Lie}(\mathscr{P})$ with the canonical induced parabolic structure on the divisor $D$ and denote this parabolic subbundle by $\operatorname{Lie}(\mathscr{P})_{*}$.

Definition 6.2. We say that the parahoric torsor $(\mathscr{E}, \boldsymbol{\theta})$ with arbitrary real weights $\boldsymbol{\theta} \in \mathscr{A}_{T}^{m}$ is semistable (respectively, stable) as a $\mathscr{G}_{\boldsymbol{\theta}, X}$-torsor if for every generic parabolic reduction datum as above,

$$
\text { par.deg } \left.\left(\operatorname{Lie}(\mathscr{P})_{*}\right) \leq 0 \text { (respectively, par.deg }\left(\operatorname{Lie}(\mathscr{P})_{*}\right)<0\right) .
$$

6.2. Second definition. Now assume that $(\mathscr{E}, \boldsymbol{\theta})$ is a parahoric torsor with rational weights. Let $P_{K} \subset \mathscr{G}_{K}$ be a maximal parabolic subgroup of the generic fiber $\mathscr{G}_{K}$ of $\mathscr{G}_{\Omega, X}$. Let $\chi: P_{K} \longrightarrow \mathbb{G}_{m, K}$ be a strictly anti-dominant character of the parabolic subgroup $P_{K}$. Therefore, the associated line bundle on $\mathscr{G}_{K} / P_{K}$ is ample. Since the quotient map $\mathscr{E}_{K} \longrightarrow \mathscr{E}_{K} / P_{K}$ defines a principal $P_{K}$-bundle, it follows that $\chi$ defines a line bundle $L_{\chi}$ on $\mathscr{E}_{K} / P_{K}=$ $\mathscr{E}_{K}\left(\mathscr{G}_{K} / P_{K}\right)$. For any reduction of structure group

$$
s_{K}: X \backslash D \longrightarrow \mathscr{E}_{K}\left(\mathscr{G}_{K} / P_{K}\right),
$$

we have the pulled back line bundle $s_{K}^{*}\left(L_{\chi}\right)$ on $X \backslash D$. This line bundle $s_{K}^{*}\left(L_{\chi}\right)$ extends $X$ by the following proposition.

Proposition 6.3 ([BSS, Proposition 6.3.1]). Let $(\mathscr{E}, \boldsymbol{\theta})$ be a parahoric torsor with rational weights. Let $s_{K}$ be a generic reduction of structure group of $\mathscr{E}_{K}$ to $P_{K}$. Then the line bundle $s_{K}^{*}\left(L_{\chi}\right)$ on $X \backslash D$ has a canonical extension $L_{\chi}^{\theta}$ to $X$ as a parabolic line bundle.

Definition 6.4 ([BSS, Definition 6.3.4]). A parahoric torsor $(\mathscr{E}, \boldsymbol{\theta})$ with rational weights is called stable (respectively, semistable) if for every maximal parabolic $\mathscr{P}_{K} \subset \mathscr{G}_{K}$, for all strictly anti-dominant character $\chi$ of $\mathscr{P}_{K}$, and for every reduction of structure group $s_{K}$ as above,

$$
\text { par.deg } \left.\left(L_{\chi}^{\boldsymbol{\theta}}\right)<0 \text { (respectively, par.deg }\left(L_{\chi}^{\boldsymbol{\theta}}\right) \leq 0\right) \text {. }
$$

We observe that the two definitions, namely Definition 6.2 and Definition 6.4, are equivalent when the weights are rational; its proof is identical to the proof of [Ra, Lemma 2.1].

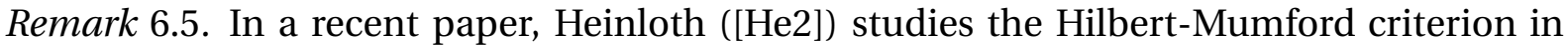
terms of algebraic stacks. The point of view developed there allows natural choices of test objects for the verification of stability, leading to criteria for the existence of separated coarse moduli spaces.

Let $\mathscr{E}$ is a $\mathscr{G}$-torsor for a parahoric group scheme $\mathscr{G}$ and let $\mathscr{B} \subset A u t_{\mathscr{G}}(\mathscr{E})$. Let $\mathscr{E}_{\mathscr{B}}$ be a reduction of structure group to $\mathscr{B}$. To a character $\chi$ of $\mathscr{B}$, one can then associate a line bundle $\mathscr{E}_{\mathscr{B}}(\lambda)$ on $X$. In the language of Section 6.2 this will be a parabolic line bundle. In $\left[\mathrm{He} 2\right.$, Section 3.5] the classical notion of a parabolic degree of $\mathscr{E}_{\mathscr{B}}(\lambda)$ is recovered in the new setting which leads to the definition of stability as in Section6.2. An advantage with his new definition is that it works in positive characteristics as well and moreover, the parahoric group scheme $\mathscr{G}$ need not be assumed to be generically split as is done in the present paper. 


\section{7. (SEMI)STABILITY AND POLYSTABILITY UNDER VARIATION OF WEIGHTS}

Let $\mathscr{V}$ be the category of all quasi-parahoric $\mathscr{G}_{\boldsymbol{\theta}, X}$-torsors along $D$ with weights $\boldsymbol{\theta}$ varying in $\mathscr{A}_{T}^{m}$. For convenience, we shall work with a single parabolic point $P \in X$. The generalization to finitely many points follows without any difficulty.

Let $r$ be the rank of $\mathscr{E}(\mathfrak{g})_{*}$. The degree of the underlying vector bundle is denoted by $d_{1}$. Note that its parabolic degree is 0 because $\mathfrak{g}=\mathfrak{g}^{*}$ as $G$-modules. Let $\mathscr{V}_{\mathfrak{g}}$ denote the space of all parabolic vector bundles such that

- the rank is the fixed integer $r$,

- the quasi-parabolic structure at $P$ is given by that of $\mathscr{E}(\mathfrak{g})_{*}$,

- the degree of the underlying vector bundle is $d_{1}$,

- the parabolic degree is zero, and

- the parabolic weights $0 \leq \alpha_{1}<\alpha_{2}<\cdots<\alpha_{r^{\prime}}<1$ are not fixed, but the length $r^{\prime}$ and the multiplicities $m_{1}, \cdots, m_{r^{\prime}}$ are fixed.

Take any $V_{*} \in \mathscr{V}_{\mathfrak{g}}$. For a subbundle $W$ of $V$, if $n_{k}$ for each $1 \leq k \leq r^{\prime}$ denote the multiplicity of $\alpha_{k}$ for the parabolic structure on $W$ induced by $\mathscr{V}_{\mathfrak{g}}$, the condition for $\alpha_{*}$-stability of $V_{*}$ is

$$
\text { degree }(W)+\sum_{k \leq r^{\prime}} n_{i_{k}} \alpha_{i_{k}}<0
$$

for all subbundles $W$ of $V$; for semistability the strict inequality is replaced by inequality. Let $\chi(V, W, \alpha)$ denote the left hand side of (7.1).

There exists a constant $C_{2} \geq 0$ such that if $\operatorname{degree}(W)>C_{2}$, then $\chi(V, W, \alpha) \geq 0$ for all $\alpha$. Thus for any $V \in \mathscr{V}_{\mathfrak{g}}$, if the underlying vector bundle $V$ admits a subbundle $W$ with degree $(W) \geq C_{2}$, then $V_{*}$ can never be parabolic semistable for any choice of weights $\alpha_{*}$. We note that the quasi-parabolic structure of $V \in \mathscr{V}_{\mathfrak{g}}$ alone determines such bundles.

7.1. Facets of a quasi-parahoric torsor. As before, $G$ is simple and simply connected.

In this subsection we shall only consider parahoric torsors $\mathscr{E} \in \mathscr{V}$ such that the quasiparabolic bundle $\mathscr{E}(\mathfrak{g})$ admits no subbundle of degree greater than $C_{2}$ :

$$
\mathscr{V}_{\mathfrak{g}}\left(C_{2}\right):=\left\{V_{*} \in \mathscr{V}_{\mathfrak{g}} \mid V \text { has no subbundle of degree greater than } C_{2}\right\} .
$$

Proposition 7.1. The set of inequalities required to verify the (semi)stability of any bundle in $\mathscr{V}_{\mathfrak{g}}\left(C_{2}\right)$ has finite cardinality.

Proof. From (7.1) we see that there exists a constant $C_{1} \leq 0$ such that

$$
\operatorname{degree}(W) \leq d_{1}+C_{1} \Rightarrow \chi(V, W, \alpha)<0 .
$$

In other words, subbundles of degree at most $C_{1}$ will never be destabilizing with respect to any inequality. Thus to check (semi)stability of $\left(\mathscr{E}(\mathfrak{g}), \theta_{\mathfrak{g}}\right)$ we may restrict ourselves to subbundles $W$ of $\mathscr{E}(\mathfrak{g})$ such that

$$
C_{1} \leq \operatorname{degree}(W) \leq C_{2} .
$$

The ranks of subbundles $W$ vary between 1 and $r-1$. Let $m_{1}, \cdots, m_{r^{\prime}}$ be the multiplicities of the quasi-parabolic structure on $\mathscr{V}_{\mathfrak{g}}$. The multiplicities $n_{i_{1}}, \cdots, n_{i_{k}}$ of the induced parabolic structure are positive integers. Thus as one varies over $\mathscr{V}_{\mathfrak{g}}$, only finitely many inequalities appear. 
If $\mathscr{E}(\mathfrak{g}) \in \mathscr{V}_{\mathfrak{g}}\left(C_{2}\right)$, it follows that to check (semi)stability of $(\mathscr{E}, \boldsymbol{\theta})$ we need to consider only finitely many inequalities corresponding to a (possibly proper) subset of the set of inequalities seen in Proposition 7.1. This is because we need to check these inequalities for subbundles which are Lie algebra bundles of certain subgroup schemes (see Definition 6.2).

We fix a maximal torus $T$ of $G$ and also fix a maximal torus $T_{\mathfrak{g}}$ of $G L(\mathfrak{g})$ such that $\operatorname{Ad}\left(T_{G}\right) \subset T_{\mathfrak{g}}$.

For every inequality,

$$
\operatorname{degree}(W)+\sum_{k \leq r^{\prime}} n_{i_{k}} \alpha_{i_{k}}<0 \text { (respectively, } \leq 0 \text { ) }
$$

in (7.1) and for every integer $c$ between $C_{1}$ (as in the proof of Proposition 7.1 ) and $C_{2}$, we associate a functional

$$
\ell_{c}: \mathscr{A}_{T_{\mathfrak{g}}} \longrightarrow \mathbb{R}
$$

as follows: for any $\eta=\left(\alpha_{1}, \cdots, \alpha_{r}\right) \in \mathscr{A}_{T_{\mathfrak{g}}}$,

$$
\ell_{c}(\eta)=\frac{c}{\operatorname{rank}(W)}+\frac{\sum_{k \leq r^{\prime}} n_{i_{k}} \alpha_{i_{k}}}{\operatorname{rank}(W)}
$$

Define $f: \mathscr{A}_{T} \longrightarrow \mathbb{R}$ by $f(\theta)=\ell_{c}(\theta(\mathfrak{g}))$. These are finitely many in number. We denote the set of these functionals by $S_{T}^{\mathscr{E}}$ (or $S_{T}$ for notational convenience). Further they are defined over rationals, since clearly the definition of $f$ only involves rational numbers and the map $\mathscr{A}_{T} \longrightarrow \mathscr{A}_{T_{\mathfrak{g}}}$ is defined over rationals and is linear. For any functional in $f$ in $S_{T}$, define the $f$-wall in $\mathscr{A}_{T}$ as

$$
W_{f}:=\left\{x \in \mathscr{A}_{T} \mid f(x)=0\right\} .
$$

The collection $\left\{W_{f}\right\}_{f \in S_{T}^{\mathscr{E}}}$ will be called the walls of $\mathscr{E}$.

Definition 7.2. Fix a quasi-parahoric torsor $\mathscr{E}$. For any $\theta \in \mathscr{A}_{T}$, let

$$
S_{1}^{\theta}=\left\{f \in S_{T}^{\mathscr{E}} \mid x \in W_{f}\right\} .
$$

Let $\mathscr{H}_{n}=\left\{x \in \mathscr{A}_{T}|\quad| S_{1}^{\theta} \mid=n\right\}$. Define a facet of $\mathscr{E}$ to be a connected component of $\mathscr{H}_{n}$ for some $n \geq 0$.

Thus the facets of $\mathscr{E}$ provide a decomposition

$$
\mathscr{A}_{T}=\bigsqcup_{n} \mathscr{H}_{n}
$$

of $\mathscr{A}_{T}$. Note that only finitely many $n$ appear here.

For any weight $\theta \in \mathscr{A}_{T}$ there is a unique $n \geq 0$ such that $\theta \in \mathscr{H}_{n}$. Thus for any $\theta \in \mathscr{A}_{T}$, the following three are equivalent:

(1) $\theta$ does not belong to any $\mathscr{E}$-wall,

(2) $S_{1}^{\theta}$ is empty, and

(3) $\theta \in \mathscr{H}_{0}$.

The facet of $\theta$ is the unique facet of $\mathscr{E}$ containing it.

The following propositions generalize [MS, Section 2, page 217]. We note that the quasiparabolic bundles $\mathscr{E}(\mathfrak{g})$ cannot admit any subbundle of degree greater than $C_{2}$ (cf. (7.2)). 
Proposition 7.3. Let $\theta$ lie in $\mathscr{A}_{T}=Y(T) \otimes \mathbb{R}$. Then there exists an element $\theta^{\prime}$ in the rational apartment $\mathscr{A}_{\mathbb{Q}}=Y(T) \otimes \mathbb{Q}$ such that for all $\mathscr{E} \in \mathscr{V}$, the pair $(\mathscr{E}, \theta)$ is semistable (respectively, stable) if and only if $\left(\mathscr{E}, \theta^{\prime}\right)$ is semistable (respectively, stable).

Proof. Let $S_{2}^{\theta}$ be the complement of $S_{1}^{\theta}$ in $S_{T}$.

We note that $\theta$ has a strictly positive distance from each $W_{f}$, where $f \in S_{2}^{\theta}$. Let $d$ be the minimum distance if $S_{2}^{\theta}$ is nonempty and set $d$ to be $\infty$ if $S_{2}^{\theta}$ is empty. Thus in all cases $d>0$. Let $U$ be the ball in the alcove of radius $d$ around $\theta$.

Let $I$ denote the $\mathscr{E}$-facet of $\theta$. Let $I_{1}$ be connected component of $I \cap U$ containing $\theta$, where $U$ is the above ball. Now if $I_{1}$ is not reduced to a single point, then we can take a rational weight $\theta^{\prime}$ in it. If $I_{1}$ is just a point, then $\theta$ must be rational because $d>0$ and all the functionals are defined over $\mathbb{Q}$. In this case, we take $\theta^{\prime}$ to be $\theta$ itself.

Let us check that the (semi)stability conditions for $\theta$ and $\theta^{\prime}$ coincide. For each functional $J$ in $S_{T}$,

(1) if $J \in S_{1}^{\theta}$ then $J\left(\theta^{\prime}\right)=J(\theta)$, because $\theta^{\prime} \in I_{1} \subset I$, and

2. if $J \in S_{2}$ then $\operatorname{sign}\left(J\left(\theta^{\prime}\right)\right)=\operatorname{sign}(J(\theta))$, because $\theta^{\prime} \in I_{1} \subset U$.

So for $\mathscr{E}$, one has $\theta^{\prime}$-(semi)stability is equivalent to $\theta$-(semi)stability.

We return to the setting of $m$-marked points on $X$ noting that the above discussion immediately goes through for multiple marked points.

Lemma 7.4. Let $\boldsymbol{\theta} \in \mathscr{A}_{T}^{m}$. Let $\rho_{i}: G \longrightarrow \mathrm{GL}\left(V_{i}\right)$ for $i \leq m$ be finitely many representations. Then there exists $\boldsymbol{\theta}^{\prime} \in \mathscr{A}_{\mathbb{Q}}^{m}$ such that for any $\mathscr{E} \in \mathscr{V}$, and any $i \leq m$, the parahoric torsor $\left(\mathscr{E}, \boldsymbol{\theta}\left(V_{i}\right)\right)$ is stable (respectively, semistable) if and only if $\left(\mathscr{E}, \boldsymbol{\theta}^{\prime}\left(V_{i}\right)\right)$ is stable (respectively, semistable).

Proof. This is immediate from Proposition 7.3 .

The following proposition is a generalization of [MS, Proposition 2.1].

Proposition 7.5. Given any $\boldsymbol{\theta}_{0} \in \mathscr{A}_{T}^{m}$, there exists a neighborhood $U$ of $\boldsymbol{\theta}_{0}$ in $\mathscr{A}_{T}^{m}$ with the property that for all $\mathscr{E} \in \mathscr{V}$ such that $\left(\mathscr{E}, \boldsymbol{\theta}_{0}\right)$ is stable, the pair $(\mathscr{E}, \boldsymbol{\theta})$ is stable for all $\boldsymbol{\theta} \in U$.

Proof. Now $\theta$ may as well be rational. Owing to the stability condition, $f(\theta)<0$ for all $f \in S_{T}$. Thus we have $S_{2}^{\theta}=S_{T}$. Let $d$ be the minimum distance between $\theta$ and any $f$-wall. Now we take $U$ to be the ball around $\theta$ radius $d$.

Definition 7.6. A parahoric torsor $(\mathscr{E}, \boldsymbol{\theta})$ for the linear parahoric group scheme $\mathscr{G} \mathscr{L}(V)$ is called polystable if the associated parabolic vector bundle $\mathscr{E}(V)_{*}$ is polystable (i.e., a direct sum of stable parabolic bundles of parabolic degree 0 ).

It is straight-forward to check that Lemma 7.4 remains valid if stability in the lemma is substituted by polystability.

Corollary 7.7. Let $\rho: G \longrightarrow \mathrm{GL}(V)$ be a representation and $\rho_{X}: \mathscr{G} \longrightarrow \mathscr{G} \mathscr{L}(V)$ the induced homomorphism of parahoric group schemes as in (5.1). Let $\mathscr{E}$ be a $\mathscr{G}$-torsor. Then, for a weight $\boldsymbol{\theta} \in \mathscr{A}_{T}^{m}$ such that $(\mathscr{E}, \boldsymbol{\theta})$ is stable, the pair $\left(\mathscr{E}(V)_{*}, \boldsymbol{\theta}(V)\right)$ is polystable. 
Proof. By Proposition (7.5), we can assume $\theta$ is rational. For a stable equivariant principal $G$-bundle, the associated bundles are polystable. Consequently, in view of the equivalence of categories in Section 3.4, The stability of $(\mathscr{E}, \boldsymbol{\theta})$ implies that $(\mathscr{E}(V), \boldsymbol{\theta}(V)$ is polystable.

\section{CONNECTIONS ON PARAHORIC $\mathscr{G}$-TORSORS}

The main aim of this section is to define connections on a parahoric $\mathscr{G}_{\boldsymbol{\theta}, X}$-torsor.

8.1. $D_{X}-$ modules. We briefly recall the definition of $D_{X}-$ modules.

Definition 8.1. Let $X \longrightarrow S$ be a $S$-scheme. Let $d x$ denote the image of $x$ under the canonical de Rham differentiation map $d: \mathscr{O}_{X} \longrightarrow \Omega_{X / S}^{1}$. Let $\mathscr{F}$ be a coherent sheaf of $\mathscr{O}_{X^{-}}$ modules over $X$. By a $D_{X}$-module structure on $\mathscr{F}$ we mean a $\mathscr{O}_{S}$-linear homomorphism of sheaf of abelian groups $\nabla: \mathscr{F} \longrightarrow \mathscr{F} \otimes_{\mathscr{O}_{X}} \Omega_{X / S}^{1}$ satisfying Leibniz rule which says that

$$
\nabla(x f)=f \otimes d x+x \nabla(f),
$$

where $f$ and $x$ are local sections of $\mathscr{F}$ and $\mathscr{O}_{X}$ respectively.

Definition 8.2. Let $\nabla_{\mathscr{F}}: \mathscr{F} \longrightarrow \mathscr{F} \otimes \Omega_{X / S}^{1}$ and $\nabla_{\mathscr{E}}: \mathscr{E} \longrightarrow \mathscr{E} \otimes \Omega_{X / S}^{1}$ be two connections over $\mathscr{F}$ and $\mathscr{E}$ respectively. Define their tensor product $\nabla_{\mathscr{F}} \otimes \nabla_{\mathscr{E}}: \mathscr{F} \otimes \mathscr{E} \longrightarrow \mathscr{F} \otimes \mathscr{E} \otimes \Omega_{X / S}^{1}$ to be

$$
\nabla_{\mathscr{F} \otimes \mathscr{E}}(f \otimes e)=\nabla_{\mathscr{F}}(f) \otimes e+f \otimes \nabla_{\mathscr{E}}(e),
$$

where $f$ and $e$ are local sections of $\mathscr{F}$ and $\mathscr{E}$ respectively.

Similarly define $\nabla_{\text {Hom }}: \operatorname{Hom}(\mathscr{E}, \mathscr{F}) \longrightarrow \operatorname{Hom}(\mathscr{E}, \mathscr{F}) \otimes \Omega_{X / S}^{1}$ to be

$$
\nabla_{\mathrm{Hom}(\mathscr{E}, \mathscr{F})}(\Phi)(e)=\nabla_{\mathscr{F}}(\Phi(e))-\Phi\left(\nabla_{\mathscr{E}}(e)\right),
$$

where $\Phi$ and $e$ are local sections of $\operatorname{Hom}(\mathscr{E}, \mathscr{F})$ and $\mathscr{E}$ respectively.

8.2. Logarithmic connections on curves. The canonical line bundle of the smooth complex projective curve $X$ will be denoted by $K_{X}$. Fix a finite subset $D=\left\{x_{i}\right\}_{1 \leq i \leq m} \subset X$; define

$$
K_{X}(\log D)=K_{X} \otimes \mathscr{O}_{X}(D) .
$$

A logarithmic connection on a vector bundle $V \longrightarrow X$ singular on $D$ is a first order algebraic differential operator $\nabla: V \longrightarrow V \otimes K_{X}(\log D)$ satisfying the Leibniz rule.

For a point $x \in D$, the fiber $K_{X}(\log D)_{x}$ is identified with $\mathbb{C}$ using the Poincaré adjunction formula. For a logarithmic connection $(V, \nabla)$, the composition

$$
V \stackrel{\nabla}{\longrightarrow} V \otimes K_{X}(\log D) \longrightarrow\left(V \otimes K_{X}(\log D)\right)_{x} \longrightarrow V_{x},
$$

which is a $\mathbb{C}$-linear endomorphism of $V_{x}$, is called the residue of $\nabla$ at $x$ [De, page 53], and it is denoted by $\operatorname{Res}(\nabla, x)$. The monodromy of $\nabla$ around $x$ is conjugate to

$$
\exp (-2 \pi \sqrt{-1} \operatorname{Res}(\nabla, x))
$$

[De, p. 53, Théorème 1.17]. 
8.3. Connection on parabolic vector bundles. Let $V \longrightarrow X$ be a vector bundle on $X$. A quasi-parabolic structure on $V$ over $D$ is a filtration, for each $x \in D$, of subspaces

$$
V_{x}=F_{1}^{x} \supsetneq F_{2}^{x} \supsetneq \cdots \supsetneq F_{a_{x}}^{x} \supsetneq F_{a_{x}+1}^{x}=\{0\} .
$$

A parabolic structure on $V$ over $D$ is a quasi-parabolic structure as above together with real numbers

$$
0 \leq \alpha_{1}^{x}<\cdots<\alpha_{i}^{x}<\cdots<\alpha_{a_{x}}^{x}<1
$$

associated to the quasi-parabolic flags. We shall often abbreviate a parabolic vector bundle $\left(V,\left\{F_{*}^{x}, \alpha_{*}^{x}\right\}_{x \in D}\right)$ by $V_{*}$.

Definition 8.3. A connection on $V_{*}$ is a logarithmic connection $\nabla$ on $V$ such that for all $x \in D$,

- the residue $\operatorname{Res}(\nabla, x)$ is semisimple and preserves the quasi-parabolic flag at $x$, meaning $\operatorname{Res}(\nabla, x)\left(F_{i}^{x}\right) \subseteq F_{i}^{x}$ for all $i$, and

- $\operatorname{Res}(\nabla, x)\left(F_{i}^{x} / F_{i+1}^{x}\right)=\alpha_{i}^{x} \operatorname{Id}_{F_{i}^{x} / F_{i+1}^{x}}$.

A connection on $V_{*}$ induces a connection on the dual parabolic vector bundle $V_{*}^{*}$. To see this, given a logarithmic connection $\nabla$ on $V$ defining a connection on $V_{*}$, consider the logarithmic connection on $V^{*} \otimes \mathscr{O}_{X}(D)$ induced by $\nabla$. This logarithmic connection preserves the subsheaf of $V^{*} \otimes \mathscr{O}_{X}(D)$ identified with the vector bundle underlying the parabolic vector bundle $V_{*}^{*}$. The logarithmic connection on this subsheaf obtained this way defines a connection on $V_{*}^{*}$.

Let $V_{*}^{1}$ and $V_{*}^{2}$ be parabolic vector bundles with underlying vector bundles $V^{1}$ and $V^{2}$ respectively. Let $\nabla^{1}$ and $\nabla^{2}$ be connections on $V_{*}^{1}$ and $V_{*}^{2}$ respectively. Consider the logarithmic connection on $V^{1} \otimes V^{2} \otimes \mathscr{O}_{X}(D)$ induced by $\nabla^{1}$ and $\nabla^{2}$. It preserves the subsheaf of $V^{1} \otimes V^{2} \otimes \mathscr{O}_{X}(D)$ corresponding to the parabolic tensor product $V_{*}^{1} \otimes{ }^{p} V_{*}^{2}$. The logarithmic connection on this subsheaf obtained this way defines a connection on $V_{*}^{1} \otimes^{p} V_{*}^{2}$.

8.4. Lie connection on a principal $G$-bundle. For a principal $G$-bundle $E \longrightarrow X$, let $E(\mathfrak{g})=$ $E_{G} \times{ }^{G} \mathfrak{g}$ be its adjoint bundle. The fibers of $E(\mathfrak{g})$ are equipped with a Lie bracket structure $[.,]:. E(\mathfrak{g}) \otimes E(\mathfrak{g}) \longrightarrow E(\mathfrak{g})$ induced by the Lie algebra structure of $\mathfrak{g}$.

Definition 8.4. A Lie connection on $E$ is a connection

$$
\nabla: E(\mathfrak{g}) \longrightarrow E(\mathfrak{g}) \otimes \Omega_{X}^{1}
$$

such that following diagram is commutative

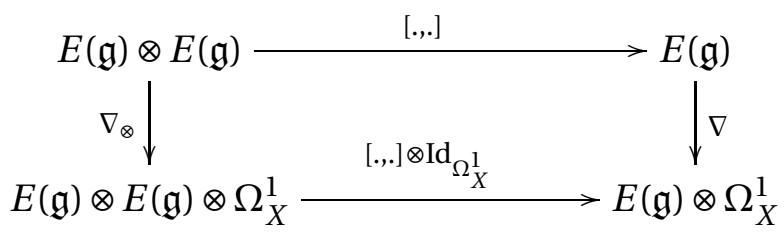

where $\nabla_{\otimes}$ is the connection on $E(\mathfrak{g}) \otimes E(\mathfrak{g})$ induced by $\nabla$.

The above commutativity condition means that the section of $E(\mathfrak{g}) \otimes(E(\mathfrak{g}) \otimes E(\mathfrak{g}))^{*}$ given by the Lie bracket operation on $E(\mathfrak{g})$ is flat with respect to the connection on $E(\mathfrak{g}) \otimes(E(\mathfrak{g}) \otimes$ $E(\mathfrak{g}))^{*}$ induced by $\nabla$. 
A connection on $E_{G}$ produces a Lie connection on $E_{G}$. Therefore, we get a map from the space of all connections on $E_{G}$ to the space of all Lie connection on $E_{G}$. Since $G$ is semisimple, the adjoint homomorphism $G \longrightarrow \mathrm{GL}(\mathfrak{g})$ has finite kernel and its image is the connected component, containing the identity element, of the group of all automorphisms of the Lie algebra $\mathfrak{g}$. From this it follows that the above map from the space of all connections on $E_{G}$ to the space of all Lie connection on $E_{G}$ is a bijection. This fact motivates the definition of a connection on a parahoric torsor.

8.5. Connection on parahoric $\mathscr{G}$-torsors. We refer to Section 5.3 for the notation. Take any $\mathscr{G}=\mathscr{G}_{\boldsymbol{\theta}, X}$.

Definition 8.5. A connection on a $\mathscr{G}$-torsor $\mathscr{E}$ is a logarithmic parabolic connection (see Definition 8.3$) \nabla$ on $\mathscr{E}(\mathfrak{g})$ satisfying the condition that the section of $\operatorname{Hom}^{p}(\mathscr{E}(\mathfrak{g}) \otimes p$ $\mathscr{E}(\mathfrak{g}), \mathscr{E}(\mathfrak{g}))$ given by the homomorphism in (5.3) is flat with respect to the connection $\nabla_{\text {hom }}$ on the parabolic vector bundle $\operatorname{Hom}^{p}\left(\mathscr{E}(\mathfrak{g}) \otimes^{p} \mathscr{E}(\mathfrak{g}), \mathscr{E}(\mathfrak{g})\right)$ induced by $\nabla$.

8.6. A Tannakian description of connection. Let $M$ be a smooth complex variety. Let $G$ be a complex reductive algebraic group and $E_{G} \longrightarrow M$ a principal $G$-bundle. Take any pair $(H, f)$, where $H$ is a complex algebraic group and $f: G \longrightarrow H$ an algebraic homomorphism such that corresponding homomorphism of Lie algebras $d f: \operatorname{Lie}(G) \longrightarrow \operatorname{Lie}(H)$ is injective. Let $E_{H}:=E_{H} \times{ }^{f} H \longrightarrow M$ be the principal $H$-bundle obtained by extending the structure group of $E_{G}$ using $f$. Let

$$
\widetilde{f}: E_{G} \longrightarrow E_{H}
$$

be the natural morphism. A connection on $E_{G}$ induces a connection on $E_{H}$. The converse is also true. To see this, fix a $G$-equivariant splitting

$$
\sigma: \operatorname{Lie}(H) \longrightarrow \operatorname{Lie}(G),
$$

meaning $\sigma \circ d f=\operatorname{Id}_{\operatorname{Lie}(G)}$ (such a splitting exists because $G$ is reductive). If $D$ is a $\operatorname{Lie}(H)-$ valued 1 -form on $E_{H}$ defining a connection on $H$, then $\sigma \circ \widetilde{f}^{*} D$ is a connection on $E_{G}$. If $D_{0}$ is a connection on $E_{G}$ and $D$ the connection on $E_{H}$ induced by $D_{0}$, then the connection $\sigma \circ \widetilde{f}^{*} D$ on $E_{G}$ coincides with $D_{0}$. Indeed, this follows immediately from the fact that $\sigma \circ$ $d f=\operatorname{Id}_{\operatorname{Lie}(G)}$.

Therefore, the map from connections on $E_{G}$ to connections on $E_{H}$ is injective. The image of this map from connections on $E_{G}$ admits a group theoretic description. This will be explained below.

We remove the assumption that the algebraic group $G$ is reductive. As before $f: G \longrightarrow H$ to be any algebraic homomorphism such that $d f$ is injective.

A theorem of C. Chevalley (see $[\mathrm{Hu}$, p. 80]) says that there is a finite dimensional left $H$-module

$$
\rho: H \longrightarrow \mathrm{GL}(W)
$$

and a complex line $\ell \subset W$ such that $f(G)$ is exactly the isotropy subgroup, of the point in the projective space $P(W)$ representing the line $\ell$, for the action of $H$ on the projective space $P(W)$ of lines in $W$ induced by the action of $H$ on $W$. Let

$$
E_{W}:=E_{H} \times{ }^{H} W \longrightarrow M
$$

be the vector bundle associated to $E_{H}$ for the $H$-module $W$. For a connection $D$ on $E_{H}$, the connection on $E_{W}$ induced by $D$ will be denoted by $D_{W}$. Note that $E_{W}$ is identified with the 
vector bundle associated to $E_{G}$ for the action $\rho \circ f$ of $G$ on $W$. The condition on $\ell$ implies that the action of $G$ on $W$ preserves it. Let $E_{\ell} \subset E_{W}$ be the line subbundle associated to the $G$-submodule $\ell \subset W$.

A connection $D$ on $E_{H}$ is induced by a connection on $E_{G}$ if and only if the corresponding connection $D_{W}$ on $E_{W}$ preserves the above line subbundle $E_{\ell} \subset E_{W}$. This characterizes the connections on $E_{H}$ that are induced by connections on $E_{G}$.

We recall that the Tannakian theory involves describing properties of principal bundles in terms of properties of associated vector bundles. For a Tannakian description of connections on $E_{G}$, take $H=\operatorname{GL}(V)$, so $V$ is a finite dimensional $G$-module. Let

$$
E_{V}:=E_{G} \times{ }^{f} V \longrightarrow M
$$

be the vector bundle associated to $E_{G}$ for the $G$-module $V$. From the above observation we know that a connection on $E_{G}$ is a connection $D$ on the vector bundle $E_{V}$ such that the connection on the vector bundle $E_{W}$ induced by $D$ preserves the line subbundle $E_{\ell} \subset E_{W}$.

\section{CONNECTiOns on $(\Gamma, G)$-BUNDLES AND RATIONAL WEIGHTS}

Let $F$ be a principal $G$-bundle on a curve $Y$ with adjoint bundle

$$
\operatorname{Ad}(F)=F(G)=F \times{ }^{G} G,
$$

where $G$ acts on itself by conjugation. Given a principal $G$-bundle $E$ on $Y$, define the "twisting" by $F$

$$
E \times{ }^{G} F^{o p}:=\left(E \times{ }_{Y} F\right) / \sim,
$$

where the equivalence relation identifies all pairs $(e, f),(e z, f z) \in E \times{ }_{Y} F$, with $z \in G$. Consider the map

$$
\xi: E \times_{Y} F \times_{Y} F \times G \longrightarrow E \times_{Y} F,\left(e, f, f z, z_{1}\right) \longmapsto\left(e z z_{1}, f z\right),
$$

where $(e, f) \in E \times_{Y} F$ and $z, z_{1} \in G$. There is a unique map

$$
\widehat{\xi}:\left(E \times{ }^{G} F^{o p}\right) \times_{Y} F(G) \longrightarrow E \times{ }^{G} F^{o p}
$$

such that the following diagram is commutative

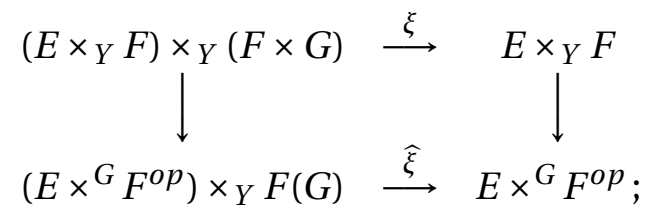

recall that $E \times{ }^{G} F^{o p}$ and $F(G)$ are quotients of $E \times{ }_{Y} F$ and $F \times G$ respectively. This map $\widehat{\xi}$ makes $E \times{ }^{G} F^{o p}$ a $F(G)$-torsor on $Y$. Let $\alpha_{F}$ be the map from the space of principal $G$-bundles to the space of $F(G)$-torsors on $Y$ defined by $E \longmapsto E \times{ }^{G} F^{o p}$. This $\alpha_{F}$ is an equivalence of categories.

Consider the adjoint action of $G$ on $\operatorname{Lie}(G)=\mathfrak{g}$. Let $\operatorname{ad}(F)=F \times{ }^{G} \mathfrak{g} \longrightarrow Y$ be the associated adjoint vector bundle. We note that $\operatorname{ad}(F)$ is the Lie algebra bundle associated to the group scheme $F(G)$.

Let $\nabla_{0}$ be a connection $F$. Using $\nabla_{0}$ we will define connections on a $F(G)$-torsor. 
The connection $\nabla_{0}$ induces a connection on every fiber bundle associated to $F$. In particular, it produces a connection on $F(G)$; this connection on $F(G)$ given by $\nabla_{0}$ will be denoted by $\nabla_{0}^{G}$. The kernel of the differential $d \pi: T F(G) \longrightarrow \pi^{*} T Y$ of the map $\pi$ in (9.1) is identified with $\pi^{*} \operatorname{ad}(F)$. So the above connection $\nabla_{0}^{G}$ on $F(G)$ gives a homomorphism

$$
\nabla_{0}^{G}: T F(G) \longrightarrow \pi^{*} \operatorname{ad}(F) .
$$

Take any $F(G)$-torsor $\varphi: E \longrightarrow Y$. Consider the action $E \times F(G) \longrightarrow E$ of $F(G)$ on $E$. Let

$$
\delta: T E \oplus \varphi^{*} T F(G) \longrightarrow T E
$$

be the differential of this map giving the action. Consider the differential of $\varphi$

$$
d \varphi: T E \longrightarrow \varphi^{*} T Y .
$$

Let

$$
T_{\varphi}:=\operatorname{kernel}(d \varphi) \subset T E
$$

be the relative tangent bundle for the projection $\varphi$. The action of $F(G)$ on $E$ identifies $T_{\varphi}$ with $\varphi^{*} \operatorname{ad}(F)$.

A connection on a $F(G)$-torsor $\varphi: E \longrightarrow Y$ is a holomorphic homomorphism of vector bundles over $Y$

$$
\beta: T E \longrightarrow \varphi^{*} \operatorname{ad}(F)=\operatorname{ad}\left(\varphi^{*} F\right)
$$

such that

(1) the restriction of $\beta$ to $T_{\varphi}$ coincides with the above identification of $T_{\varphi}$ with $\varphi^{*} \operatorname{ad}(F)$, and

(2) for the homomorphism $\delta$ in (9.3),

$$
\delta\left(\operatorname{kernel}(\beta) \oplus \varphi^{*} \operatorname{kernel}\left(\nabla_{0}^{G}\right)\right) \subset \operatorname{kernel}(\beta),
$$

where $\nabla_{0}^{G}$ is the homomorphism in (9.2).

Note that the above definition of a connection on $E$ depends on $\nabla_{0}$.

If $F$ is the trivial principal $G$-bundle $Y \times G$, then $F(G)=Y \times G$, and a $F(G)$-torsor is in fact a principal $G$-bundle on $Y$. If we choose $\nabla_{0}$ to be the trivial connection on $Y \times G$, then connections on a $F(G)$-torsor are same as connections on the corresponding principal $G-$ bundle.

The following lemma is straight-forward to check.

Lemma 9.1. Given a $F(G)$-torsor $\varphi: E \rightarrow Y$, a homomorphism

$$
\beta: T E \longrightarrow \varphi^{*} \operatorname{ad}(F)=\operatorname{ad}\left(\varphi^{*} F\right)
$$

defines a connection on $E$ if and only if

$$
\delta^{*} \beta=\beta \oplus \nabla_{0}^{G}
$$

on $T E \oplus \varphi^{*} T F(G)$, where $\delta$ is constructed in (9.3) and $\nabla_{0}^{G}$ is the homomorphism in (9.2).

Proposition 9.2. Twisting by $F$ defines an equivalence between principal G-bundles equipped with a connection and $F(G)$-torsors equipped with a connection. 
Proof. Let $E$ be a principal $G$-bundle on $Y$. Let $D$ be a connection on $E$. Consider $D$ as a $\mathfrak{g}$-valued 1-form on $E$. Let $D^{\prime}$ denote the $\mathfrak{g}$-valued 1-form on $F$ corresponding to the connection $\nabla_{0}$ on $F$. So $\left(D, D^{\prime}\right)$ is a $\mathfrak{g}$-valued 1 -form on the fiber product $E \times_{Y} F$. The pullback of $\operatorname{ad}(F)$ to $F$ is identified with the trivial vector bundle $F \times \mathfrak{g} \longrightarrow F$. Therefore, $\left(D, D^{\prime}\right)$ defines a 1-form with values in the pullback of $\operatorname{ad}(F)$ to $E \times{ }_{Y} F$. This form on $E \times{ }_{Y} F$ descends to the quotient $F(G)$-torsor $E \times{ }^{G} F^{o p}$ as a 1 -form with values in the pullback of $\operatorname{ad}(F)$ to $E \times{ }^{G} F^{o p}$. It is straight-forward to check that this form defines a connection on the $F(G)$-torsor corresponding to $E$.

Conversely, let $\beta$ be a connection on a $F(G)$-torsor $\varphi: E \longrightarrow Y$. Consider the pullback $\beta^{\prime}$ of $\beta$ to $E \times{ }_{Y} F$ as a 1-form with values in the pullback of $\operatorname{ad}(F)$. As noted above, the pullback of $\operatorname{ad}(F)$ to $E \times{ }_{Y} F$ is identified with the trivial vector bundle with fiber $\mathfrak{g}$. So $\beta^{\prime}$ is a 1-form on $E \times{ }_{Y} F$ with values in $\mathfrak{g}$. Let $D^{\prime}$ be the pullback of the connection form $D$ to $E \times{ }_{Y} F$. Then $\beta^{\prime}-D^{\prime}$ descends to $E$ by the projection $E \times{ }_{Y} F \longrightarrow E$, and this descended form defines a connection on the principal $G$-bundle $E$.

The above two constructions are evidently inverses of each other.

Assume that $Y$ is equipped with the action of a finite group $\Gamma$. A $\Gamma$-connection on a $(\Gamma, G)$-bundle $E$ on $Y$ is a connection on $E$ which is preserved by the action of $\Gamma$.

Proposition 9.3. Let $E \longrightarrow Y$ be a $(\Gamma, G)$-bundle on some Galois cover $p: Y \longrightarrow X$ with Galois group $\Gamma$. Let $\mathscr{E}$ be the parahoric torsor on $X$ with rational weights corresponding to $E$. Then there is a natural bijection between the connections on $\mathscr{E}$ and the $\Gamma$-connections on $E$.

Proof. This follows from the fact that the connections on a $\Gamma$-equivariant vector bundle on $Y$ are in bijection with the connections on the corresponding parabolic vector bundle on $X$.

\section{Flat UNITARY CONNECTIONS ON PARAHORIC TORSORS AND STABILITY}

\subsection{Polystable parahoric torsors.}

Lemma 10.1. Let $V_{*}$ be a polystable parabolic vector bundle of parabolic degree zero with real weights $\theta$. Then the parabolic vector bundle $\left(V_{*}\right)^{\otimes m} \otimes^{p}\left(\left(V_{*}\right)^{*}\right)^{\otimes n}$ is also polystable.

Proof. A parabolic vector bundle of parabolic degree zero is polystable if and only if it is given by a unitary representation of $\pi_{1}(X \backslash D)$, where $D$ is the parabolic divisor [MS], [Biq]. Since $V_{*}$ is polystable, it is given by a representation $\rho$ of $\pi_{1}(X \backslash D)$. The parabolic vector bundle $\left(V_{*}\right)^{\otimes m} \otimes{ }^{p}\left(\left(V_{*}\right)^{*}\right)^{\otimes n}$ is given by the representation $\rho^{\otimes m} \otimes(\bar{\rho})^{\otimes n}$. This implies that $\left(V_{*}\right)^{\otimes m} \otimes^{p}\left(\left(V_{*}\right)^{*}\right)^{\otimes n}$ is polystable.

Corollary 10.2. Take $V_{*}$ as in Lemma 10.1, Take any homomorphism

$$
\rho: \operatorname{GL}(r, \mathbb{C}) \longrightarrow \operatorname{GL}(N, \mathbb{C}),
$$

where $r$ is the rank of $V_{*}$. Let $W_{*}$ be the parabolic vector bundle associated to $V_{*}$ for $\rho$. Then $W_{*}$ is also polystable.

Proof. Consider $\mathbb{C}^{N}$ as a GL $(r, \mathbb{C})$-module using $\rho$ and the standard representation of $\operatorname{GL}(N, \mathbb{C})$. This $\mathrm{GL}(r, \mathbb{C})$-module $\mathbb{C}^{N}$ is a direct summand of a direct sum of $\mathrm{GL}(r, \mathbb{C})$ modules of the form $\left(\mathbb{C}^{r}\right)^{\otimes m_{i}}\left(\left(\mathbb{C}^{r}\right)^{*}\right)^{\otimes n_{i}}$ [DMOS, p. 40, Proposition 3.1(a)]. Therefore, from 
Lemma 10.1 we conclude that $W_{*}$ is a direct summand of a polystable parabolic vector bundle of parabolic degree zero. Hence $W_{*}$ is polystable.

We define polystability for parahoric torsors.

Definition 10.3. Let $\mathscr{G}_{\theta, X} \longrightarrow X$ be a Bruhat-Tits group scheme with generic fiber $G$. A parahoric $\mathscr{G}_{\boldsymbol{\theta}, X}$-torsor $\mathscr{E}$ with real weights $\boldsymbol{\theta}$ is said to be polystable if for every representation $\rho: G \longrightarrow \mathrm{GL}(V)$, the corresponding parabolic vector bundle $(\mathscr{E}, \boldsymbol{\theta}(V))$ is polystable in the sense of Definition 7.6 .

10.2. Polystable parahoric torsors from unitary representations. In this subsection we will first assume that $D=\{x\}$ is a single point. The multi-point case is actually a straightforward generalization.

The complement $X \backslash\{x\}$ would be denoted by $Y$. For a base point $y_{0} \in Y$, set $\Gamma=$ $\pi_{1}\left(Y, y_{0}\right)$. Choose an analytic disc $U \subset X$ around $x$ such that $y_{0} \in U$. The inclusion of $U \backslash\{x\}$ in $Y$ produces an inclusion $\pi_{1}\left(U \backslash\{x\}, y_{0}\right) \hookrightarrow \Gamma$. Using the orientation of $U \backslash\{x\}$, the group $\pi_{1}\left(U \backslash\{x\}, y_{0}\right)$ gets identified with $\mathbb{Z}$. The element of $\pi_{1}\left(U \backslash\{x\}, y_{0}\right)$ corresponding to $1 \in \mathbb{Z}$ will be denoted by $\gamma$.

We now recall a description of the set of conjugacy classes in a compact semisimple and simply connected group in terms of the Weyl alcove (see [BS, page 9]). Let $K_{G} \subset G$ be a fixed maximal compact subgroup and $T$ a fixed maximal torus in $K_{G}$. The corresponding Weyl group in the quotient $N_{G}(T) / T$. The set of conjugacy classes of element in $K_{G}$ gets identified with the $T / W$ which is in fact the Weyl alcove because any element of $K_{G}$ is conjugate to an element in the maximal torus up to an element of the Weyl group (cf. [Mor, page 151]). Given any $t \in K_{G}$, let $\theta_{t}$ denote the point in the Weyl alcove corresponding to $t$.

Given any homomorphism

$$
\rho: \Gamma \longrightarrow K_{G}
$$

let $E_{\rho}$ be the flat principal $G$-bundle on $Y$ associated to it. To construct $E_{\rho}$, let $\left(\widetilde{Y}, \widetilde{y}_{0}\right)$ be the pointed universal cover of $Y$ corresponding to the base point $y_{0}$; note that $\Gamma$ acts on $\widetilde{Y}$. Identify two points $\left(y_{1}, g_{1}\right),\left(y_{2}, g_{2}\right) \in \widetilde{Y} \times G$ if there is an element $\gamma \in \Gamma$ such that $\left(y_{2}, g_{2}\right)=\left(y_{1} \gamma, \rho\left(\gamma^{-1}\right) g_{2}\right)$. The quotient of $\widetilde{Y} \times G$ is a principal $G$-bundle on $Y$, which is denoted by $E_{\rho}$; the right translation action of $G$ on $\widetilde{Y} \times G$ produces an action of $G$ on $E_{\rho}$. The flat connection on the trivial principal $G$-bundle $\widetilde{Y} \times G \longrightarrow \widetilde{Y}$ descends to a flat connection on $E_{\rho}$. For any $h \in K_{G}$, the map

$$
\widetilde{Y} \times G \longrightarrow \tilde{Y} \times G, \quad(y, z) \longmapsto(y, \rho(h) z)
$$

descends to an isomorphism

as flat principal $G$-bundles on $Y$.

$$
E_{h \rho h^{-1}} \stackrel{\sim}{\longrightarrow} E_{\rho}
$$

Let

$$
t=\rho(\gamma) \in K_{G}
$$

be the image of $\gamma$. Since $h . \rho \cdot h^{-1}(\gamma)=h t h^{-1}$ for all $h \in K_{G}$, there is the map

$$
\operatorname{Hom}\left(\Gamma, K_{G}\right) / K_{G} \longrightarrow \mathscr{A}, \quad \rho \longmapsto \theta_{t} .
$$

After conjugating $\rho$ by an element of $K_{G}$, we may assume that $t$ belongs to a fixed maximal torus $T$ of $K_{G}$. 
Let $\mathbf{t} \in \operatorname{Lie}(T)$ be such that

$$
\exp (-2 \pi \sqrt{-1} \mathbf{t})=t,
$$

where exp denotes the exponential map on the Lie algebra Lie $(T)$. Consider the trivial principal $G$-bundle $(U \backslash\{x\}) \times G$ over $U \backslash\{x\}$. The trivial connection on it given by this trivialization will be denoted by $d_{0}$. On $(U \backslash\{x\}) \times G$, we now have the flat connection

$$
\widehat{d}=d_{0}+\frac{\mathbf{t} d z}{z},
$$

where $z$ is a holomorphic coordinate function on $U$ with $z(x)=0$.

Restrict the representation $\rho$ to the subgroup $\pi_{1}\left(U \backslash\{x\}, y_{0}\right)$. This produces a flat principal $G$-bundle $E_{\rho}(\infty) \longrightarrow U \backslash\{x\}$. Note that

$$
\left.E_{\rho}\right|_{U \backslash\{x\}} \simeq E_{\rho}(\infty)
$$

as flat principal $G$-bundles. Both the flat principal $G$-bundles in (10.4) are isomorphic to the flat principal $G$-bundle $((U \backslash\{x\}) \times G, \widehat{d})$ constructed in (10.3). This is because all of them have the same residue, namely $\mathbf{t}$, at $x$. Recall that the residue determines the conjugacy class of the monodromy (see (8.4)).

To the element $t \in K_{G} \cap T$, we have the associated conjugacy class $\theta_{t} \in \mathscr{A}$, and hence by Bruhat-Tits theory have a group scheme $\mathscr{G}_{\theta_{t}}$ on a formal neighborhood $\widehat{U}=\operatorname{Spec} \mathbb{C}[[t]]$ of $x$. This group scheme $\mathscr{G}_{\theta_{t}}$ is the trivial group scheme Spec $\mathbb{C}((t)) \times G$ over $\widehat{U} \backslash x=\operatorname{Spec} \mathbb{C}((t))$. Therefore, we can extend $\mathscr{G}_{\theta_{t}}$ uniquely to $X$ by setting it to be the trivial group scheme $Y \times G$ over $Y$ [BS, Section 5.2, page 28]. We denote this group scheme on $X$ by $\mathscr{G}_{t, X}$.

We observe that there is a morphism $\widehat{U} \hookrightarrow U$, where one identifies the formal power series ring with the completion of the convergent power series ring. Consider the trivial $\mathscr{G}_{\theta_{t}}$-torsor on $\widehat{U}$ which we denote by $P_{t}$. Note that the connection on $E_{\rho}(\infty)$ restricts to a natural connection on $\left.P_{t}\right|_{\widehat{U} \backslash\{x\}}$.

We now patch together (using for example [BS, 5.2.3]) the trivial $\mathscr{G}_{\theta_{t}}$-torsor $P_{t}$ on $\widehat{U}$ and the principal $G$-bundle $E_{\rho}$ over $Y$ along the intersection $\widehat{U} \backslash\{x\}=\operatorname{Spec} \mathbb{C}((t))$ such that the patching is connection preserving; as noted above, on Spec $\mathbb{C}((t))$, both the principal $G-$ bundles are the trivial principal $G$-bundle $\operatorname{Spec} \mathbb{C}((t)) \times G$ equipped with the connection $\widehat{d}$.

The above construction is summarized in the following proposition.

Proposition 10.4. Given any homomorphism $\rho: \Gamma \longrightarrow K_{G}$, and any $\boldsymbol{t} \in \operatorname{Lie}(T)$ satisfying (10.2), the flat principal $G$-bundle on $Y$ has a canonical extension to a $\mathscr{G}_{t, X}$-torsor over $X$.

It should be clarified that the $\mathscr{G}_{t, X}$-torsor in Proposition (10.4) depends on the choice of $\mathbf{t}$ (a branch of the logarithm), while the isomorphism class of the Bruhat-Tits group scheme $\mathscr{G}_{t, X}$ depends only on the conjugacy class $[t]$ of $t$. For $G=\mathrm{GL}(r, \mathbb{C})$, if the logarithm $\mathbf{t}$ is chosen as done in [MS] (meaning $\mathbf{t}$ is semisimple and eigenvalues are nonnegative and less than 1), then the construction in (10.4) coincides with the construction in [MS| of a parabolic vector bundle from a homomorphism $\Gamma \longrightarrow \mathrm{U}(r)$. This follows by comparing the two constructions.

The $\mathscr{G}_{t, X}$-torsor in Proposition (10.4) will be denoted by $E_{\rho}(t)$.

It may be mentioned that we may restrict the connection $\widehat{d}$ in (10.3) to $\widehat{U} \backslash\{x\} \subset U \backslash\{x\}$, where $\widehat{U}$ as before is the formal completion along $x$. A $\mathscr{G}_{t, X}$-torsor on $X$ can be trivialized 
over both $Y$ and $\widehat{U}$. Conversely, given $\mathscr{G}_{t, X}$-torsors on $Y$ and $\widehat{U}$, and an isomorphism between them over $\widehat{U} \backslash\{x\}$, we get a $\mathscr{G}_{t, X}$-torsor on $X$. Therefore, the connection over $\widehat{U} \backslash\{x\}$ is enough to construct the $\mathscr{G}_{t, X}$-torsor $E_{\rho}(t)$.

10.3. Polystable parahoric torsors and unitary representations. As before, fix a maximal compact subgroup $K_{G}$ of $G$.

Theorem 10.5. Let $(\mathscr{E}, \boldsymbol{\theta})$ be a parahoric $\mathscr{G}_{\boldsymbol{\theta}, X}$-torsor on $X$ with arbitrary real weights $\boldsymbol{\theta} \in$ $\mathscr{A}_{T}^{m}$. Then $(\mathscr{E}, \boldsymbol{\theta})$ is polystable if and only if $(\mathscr{E}, \boldsymbol{\theta})$ is given by a homomorphism from $\pi_{1}(X \backslash D)$ to $K_{G}$ as described in Section 10.2 .

Proof. First assume that $(\mathscr{E}, \boldsymbol{\theta})$ is given by a homomorphism

$$
\beta: \pi_{1}(X \backslash D) \longrightarrow K_{G} .
$$

Let $\rho: G \longrightarrow \mathrm{GL}(V)$ be any homomorphism. Fix a maximal compact subgroup $K_{\mathrm{GL}(V)}$ of $\mathrm{GL}(V)$ such that $\beta\left(K_{G}\right) \subset K_{\mathrm{GL}(V)}$. Then the parabolic vector bundle $W_{*}$ associated to $(\mathscr{E}, \boldsymbol{\theta})$ for $\rho$ is given by the homomorphism

$$
\rho \circ \beta: \pi_{1}(X \backslash D) \longrightarrow K_{\mathrm{GL}(V)} .
$$

Therefore, this associated parabolic vector bundle $W_{*}$ is polystable.

To prove the converse, assume that $(\mathscr{E}, \boldsymbol{\theta})$ is polystable. Let $\mathscr{E}(\mathfrak{g})$ and $\mathscr{E}(\mathfrak{g} \otimes \mathfrak{g})$ be the parabolic vector bundles associated to $(\mathscr{E}, \boldsymbol{\theta})$ for the $G$-modules $\mathfrak{g}$ and $\mathfrak{g} \otimes \mathfrak{g}$ respectively. From Definition 10.3 we know that both $\mathscr{E}(\mathfrak{g})$ and $\mathscr{E}(\mathfrak{g} \otimes \mathfrak{g})$ are polystable of parabolic degree zero. If $\mathscr{E}(\mathfrak{g})$ is given by a homomorphism $\beta$ from $\pi_{1}(X \backslash D)$ to a maximal compact subgroup of $\mathrm{GL}(\mathfrak{g})$, then $\mathscr{E}(\mathfrak{g} \otimes \mathfrak{g})$ is given by $\beta \otimes \beta$.

For any two parabolic vector bundles given by unitary representations of $\pi_{1}(X \backslash D)$, any homomorphism between them is given by a homomorphism of $\pi_{1}(X \backslash D)$-modules. Let

$$
\gamma: \mathscr{E}(\mathfrak{g} \otimes \mathfrak{g}) \longrightarrow \mathscr{E}(\mathfrak{g})
$$

be the homomorphism of parabolic vector bundles given by the Lie bracket $\mathfrak{g} \otimes \mathfrak{g} \longrightarrow \mathfrak{g}$. From the above statement we conclude that $\gamma$ is given by a homomorphism of $\pi_{1}(X \backslash D)$ modules. This implies that the connection on $\mathscr{E}(\mathfrak{g})$ is induced by a connection on $(\mathscr{E}, \theta)$ (see Definition 8.5). Therefore, $(\mathscr{E}, \boldsymbol{\theta})$ is given by a homomorphism from $\pi_{1}(X \backslash D)$ to $K_{G}$.

A homomorphism $\rho: \pi_{1}(X \backslash D) \longrightarrow K_{G}$ is called irreducible if $\rho\left(\pi_{1}(X \backslash D)\right)$ is not contained in some proper parabolic subgroup of $G$. A homomorphism $\rho$ is irreducible if and only if the space of invariants in $\mathfrak{g}$ for the adjoint action of $\rho\left(\pi_{1}(X \backslash D)\right)$ is the zero element.

Corollary 10.6. Let $(\mathscr{E}, \boldsymbol{\theta})$ be a parahoric $\mathscr{G}_{\boldsymbol{\theta}, X}$-torsor on $X$ with arbitrary real weights $\boldsymbol{\theta} \in$ $\mathscr{A}_{T}^{m}$. Then $(\mathscr{E}, \boldsymbol{\theta})$ is stable if and only if $(\mathscr{E}, \boldsymbol{\theta})$ is given by an irreducible homomorphism from $\pi_{1}(X \backslash D)$ to $K_{G}$ as described in Section 10.2 .

Proof. Assume that $(\mathscr{E}, \boldsymbol{\theta})$ is stable. Therefore, $(\mathscr{E}, \boldsymbol{\theta})$ is polystable. If the homomorphism $\rho: \pi_{1}(X \backslash D) \longrightarrow K_{G}$ corresponding to $(\mathscr{E}, \boldsymbol{\theta})$ has the property that $\rho\left(\pi_{1}(X \backslash D)\right)$ is contained in a proper parabolic subgroup $P$ of $G$, then the reduction of $\mathscr{E}$ to $P$ over $X \backslash D$ contradicts the stability of $(\mathscr{E}, \boldsymbol{\theta})$. Therefore, $\rho$ is irreducible.

Conversely, for a polystable $(\mathscr{E}, \boldsymbol{\theta})$, if the corresponding homomorphism $\rho: \pi_{1}(X \backslash D) \longrightarrow$ $K_{G}$ is irreducible, then the polystable parabolic vector bundle $\mathscr{E}(\mathfrak{g})$ does not admit any 
holomorphic section [Si, p. 744, Theorem 3]. Consequently, the polystable torsor $(\mathscr{E}, \boldsymbol{\theta})$ is stable.

Remark 10.7. The Corlette-Donaldson-Hitchin-Simpson correspondence between flat $G-$ bundles and $G$-Higgs bundles also extends to the parahoric case. When $G=\operatorname{GL}(n, \mathbb{C})$ this was proved by Simpson in [Si]. This result of [Si] is the key ingredient in this extension for general $G$. Using this result the question for $G$ is reduced to one on vector bundles using the adjoint representation of $G$. The approach in the present paper then goes through without any essential difficulty.

Remark 10.8. The paper $[\overline{\mathrm{BGM}}]$ considers the problem of parabolic Higgs $G$-bundle and the Corlette-Donaldson-Hitchin-Simpson correspondence on curves from a somewhat different perspective and also consider real representations.

Remark 10.9. The Atiyah-Weil criterion, $[\overline{\mathrm{At}}], \mid \mathrm{We}], \mid \mathrm{AB}]$, for the existence of a holomorphic connection on a holomorphic principal $G$-bundle generalizes to $\mathscr{G}$-torsors. The proof in $\mathrm{AB}$ has a straight-forward generalization. Similarly, the Atiyah-Krull-Schmitt reduction of a holomorphic principal $G$-bundle, [BBN2], generalizes to $\mathscr{G}$-torsors.

Remark 10.10. Theorem 10.5 evidently generalizes to the situation where $G$ is a product of simple and simply connected groups. The more general case of semisimple groups $G$ that are not simply connected is covered by using twisted bundles as in [BLS]. For a reductive group $G$, the natural map $G \longrightarrow G / Z_{0}(G) \times(G /[G, G])$ is surjective with finite kernel, where $Z_{0}(G)$ is the connected component of the center of $G$ containing the identity element. Since $G / Z_{0}(G)$ is semisimple and $/[G, G]$ is a product of copies of $\mathbb{G}_{m}$, to prove Theorem 10.5 it suffices to prove it for $\mathbb{G}_{m}$. But this was done in [Si].

10.4. The reductive case. We now indicate briefly how to extend the consideration of (semi)stability of torsors in the case when the structure group $G$ is a connected reductive algebraic group and identify it with the space of homomorphisms from $\pi$ to $K_{G}$. However, the corresponding relationship with parahoric torsors for reductive $G$ needs a closer analysis of Bruhat-Tits theory for reductive groups.

Let $S=[G, G]$ be the derived group, i.e. the maximal connected semisimple subgroup of $G$. Let $Z_{0}$ be the connected component of the center of $G$ (which is a torus) and one know that $S$ and $Z_{0}$ together generate $G$. Let $H=Z_{0} \times S$. Then in fact, $H \longrightarrow G$ is a finite covering map. It is easy to see (following [Ra, page 145]) that $(\Gamma, H)$-bundles gives rise to $(\Gamma, G)$-bundles and the stability and semistability of the associated $(\Gamma, G)$-bundles follows immediately from that of the $(\Gamma, H)$-bundles.

Thus, the problem of handling the reductive group $H$ reduces to the problem of handling the semisimple group $G$ but which is not simply connected. On the side of BruhatTits group schemes and parahoric group schemes, for a general connected reductive $G$ the existence of Bruhat-Tits group schemes are well known and would give the existence of similar group schemes on the whole of $X$. Several technical issues which one has avoided are in the setting of the Bruhat-Tits buildings. Canonical choices of apartments and alcoves which give a transparent meaning to the association of conjugacy classes with weights in the alcove would need technical modifications which led us too far afield; future considerations would therefore need a careful discussion on a "canonical" choice of apartment as for example indicated in ([Tits, page 32]). 


\section{ACKNOWLEDGEMENTS}

We are very grateful to the referee for very detailed comments. A portion of Section 1.1 was formulated by the referee. The first two authors acknowledge partial support by the J. C. Bose Fellowship. The first author thanks Department of Mathematics, Pondicherry University for its hospitality where this work was completed.

\section{REFERENCES}

[At] M.F. Atiyah, Complex analytic connections in fibre bundles, Trans. Amer. Math. Soc. 85 (1957) 181207.

[AB] H. Azad and I. Biswas, On holomorphic principal bundles over a compact Riemann surface admitting a flat connection, Math. Ann. 322 (2002), 333-346.

[BLS] A. Beauville, Y. Laszlo and C. Sorger, The Picard group of the moduli of G-bundles on a curve, Compositio Math. 112 (1998), 183-216.

[BBN1] V. Balaji, I. Biswas and D. S. Nagaraj, Principal bundles over projective manifolds with parabolic structure over a divisor, Tohoku Math. Jour. 53 (2001), 337-368.

[BBN2] V. Balaji, I. Biswas and D. S. Nagaraj, Krull-Schmidt reduction for principal bundles, Jour. Reine Angew. Math. 578 (2005), 225-234.

[BS] V. Balaji and C.S Seshadri, Moduli of parahoric $\mathscr{G}$-torsors on a compact Riemann surface, Jour. Alg. Geom. 24 (2015), 1-49. (arxiv:math.AG 1009.3485)

[Bis] I. Biswas, Parabolic bundles as orbifold bundles, Duke Math. Jour. 88 (1997), 305-325.

[Biq] O. Biquard, Fibrés Paraboliques Stables et Connexions Singulières Plates, Bull. Soc. Math. Fr. 119 (1991), 231-257.

[BGM] O. Biquard, Oscar Garcia-Prada, Ignasi Mundet i Riera, Parabolic Higgs bundles and representations of the fundamental group of a punctured surface into a real group, arXiv:1510.04207v1.

[Bo] P. Boalch, Riemann-Hilbert for tame complex parahoric connections, Transform. Gr. 16 (2011), 2750. (math arxiv DG 1003.3177).

[BLR] S. Bosch, W. Lutkebohmert and M. Raynaud, Neron models, Ergebnisse 21, Springer Verlag, BerlinNew York, 1990.

[BT1] F. Bruhat and J. Tits, Groupes réductifs sur un Corps Local, I: Données radicielles valuées, Inst. Hautes Éludes Sci. Publ. Math. 41 (1972), 5-252.

[BT2] F. Bruhat and J. Tits, Groupes réductifs sur un corps local II, Schémas en groupes, Inst. Hautes Éludes Sci. Publ. Math. 60 (1984), 197-376.

[De] P. Deligne, Équations différentielles à points singuliers réguliers, Lecture Notes in Mathematics, Vol. 163, Springer-Verlag, Berlin-New York, 1970.

[DMOS] P. Deligne, J. S. Milne, A. Ogus and K.-y. Shih, Hodge cycles, motives, and Shimura varieties, Lecture Notes in Mathematics, 900, Springer-Verlag, Berlin-New York, 1982.

[Ed] B. Edixhoven, Néron models and tame ramification, Compos. Math. 81 (1992), 291-306.

[He1] J. Heinloth, Uniformization of $\mathscr{G}$-bundles, Math. Ann. 347 (2010), 499-528.

[He2] J. Heinloth, Hilbert-Mumford stability on algebraic stacks and applications to $\mathscr{G}$-bundles on curves, arXiv:1609.06058.

[Hu] J. E. Humphreys, Linear algebraic groups, Graduate Texts in Mathematics, Vol. 21, Springer-Verlag, New York, Heidelberg, Berlin, 1987.

[La] E.Landvogt, Some functorial properties of the Bruhat-Tits building, Jour. Reine Angew. Math. 518 (2000), 213-241.

[MY] M. Maruyama and K.Yokogawa, Moduli of parabolic stable sheaves. Math. Ann. 293 (1992), 77-99.

[MS] V.Mehta and C.S. Seshadri, Moduli of vector bundles on smooth curves with parabolic structures, Math. Ann. 248 (1980), 205-239.

[Mor] J.W. Morgan, Holomorphic bundles over elliptic manifolds, ICTP Lecture Notes, Volume 1, (2000).

[Na] M. Namba, Branched coverings and algebraic functions, Pitman Research Notes in Mathematics Series, 161, Longman Scientific \& Technical, Harlow; John Wiley \& Sons, Inc., New York, 1987.

[NS] M. S. Narasimhan and C. S. Seshadri, Stable and unitary vector bundles on a compact Riemann surface, Ann. of Math. 82 (1965), 540-567. 
[No] M.V. Nori, The fundamental group scheme, Proc. Ind. Acad. Sci. Math. Sci. 91 (1982), 73-122.

[PR1] G. Pappas and M. Rapoport, Twisted loop groups and their affine flag varieties, Advances in Math 219 (2008), 118-198.

[PR2] G. Pappas and M. Rapoport, Some questions about $\mathscr{G}$-bundles on curves, Algebraic and arithmetic structures of moduli spaces (Sapporo 2007), pp. 159-171, Adv. Stud. Pure Math., vol. 58, Math. Soc. Japan, Tokyo, 2010.

[Ra] A. Ramanathan, Stable principal bundles on a compact Riemann surface, Math. Ann. 213 (1975), 129-152.

[RS] A. Ramanathan and Subramanian, Einstein-Hermitian connections on principal bundles and stability, Jour. Reine Angew. Math. 390 (1988), 21-31.

[Se1] C. S. Seshadri, Moduli of $\pi$-vector bundles over an algebraic curve, Questions On algebraic Varieties, C.I.M.E, Varenna, (1969), 139-261. (For a new corrected version see http://www.cmi.ac.in/ balaji/Publications/CSS1.19.pdf).

[Se2] C. S. Seshadri, Remarks on Parabolic Structures, Contemporary Mathematics, Volume 522, Papers in Honour of S.Ramanan, 2010.

[SGA] Séminaire de Géométrie Algèbrique du Bois-Marie SGA3, Séminaire 1962/1964 - Schémas en groupes - (SGA 3) - vol. 1 (Lecture notes in mathematics 151) (in French). Berlin; New York: SpringerVerlag.

[Si] C. T. Simpson, Harmonic bundles on noncompact curves, Jour. Amer. Math. Soc. 3 (1990), 713-770.

[Sp] T. A. Springer, Linear algebraic groups, Second edition, Progress in Mathematics, 9, Birkhäuser Boston, Inc., Boston, MA, 1998.

[TW] C. Teleman and C. Woodward, Parabolic bundles, products of conjugacy classes, and quantum cohomology, Ann. Institut Fourier 3 (2003), 713-748.

[Tits] J. Tits, Reductive groups over local fields, Proceedings of Symposia in Pure Mathematics, Vol 33, (1979), 29-69.

[We] A. Weil, Généralisation des fonctions abéliennes, Jour. Math. Pures Appl. 17 (1938), 47-87.

Chennai Mathematical Institute, Sipcot IT Park, Siruseri 603103, India

E-mail address: balaji@cmi.ac.in

School of Mathematics, Tata Institute of Fundamental Research, Homi Bhabha Road, Mumbai 400005, INDIA

E-mail address: indranil@math.tifr.res.in

Indian Institute of Science Education and Research, Mohali Knowledge city, Sector 81, SaS NAGAR, MANAULI PO 140306, INDia

E-mail address: ypandey@i isermohali .ac.in 